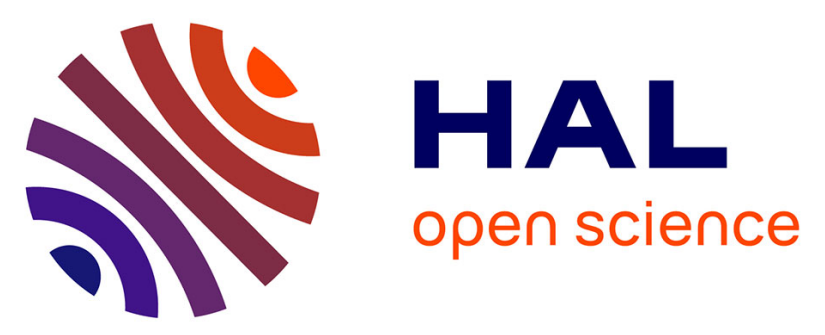

\title{
The Seismic Attenuation System (SAS) for the Advanced LIGO gravitational wave interferometric detectors
}

\author{
Alberto Stochino, Benjamin Abbot, Yoichi Aso, Mark Barton, Alessandro \\ Bertolini, Valerio Boschi, Dennis Coyne, Riccardo Desalvo, Carlo Galli, Yumei \\ Huang, et al.
}

\section{To cite this version:}

Alberto Stochino, Benjamin Abbot, Yoichi Aso, Mark Barton, Alessandro Bertolini, et al.. The Seismic Attenuation System (SAS) for the Advanced LIGO gravitational wave interferometric detectors. Nuclear Instruments and Methods in Physics Research Section A: Accelerators, Spectrometers, Detectors and Associated Equipment, 2009, 598 (3), pp.737-753. hal-01092407

\section{HAL Id: hal-01092407 https://hal.science/hal-01092407}

Submitted on 8 Dec 2014

HAL is a multi-disciplinary open access archive for the deposit and dissemination of scientific research documents, whether they are published or not. The documents may come from teaching and research institutions in France or abroad, or from public or private research centers.
L'archive ouverte pluridisciplinaire HAL, est destinée au dépôt et à la diffusion de documents scientifiques de niveau recherche, publiés ou non, émanant des établissements d'enseignement et de recherche français ou étrangers, des laboratoires publics ou privés. 


\title{
The Seismic Attenuation System (SAS) for the Advanced LIGO gravitational wave interferometric detectors
}

\author{
Alberto Stochino a,b,c,*, Benjamin Abbot ${ }^{b}$, Yoichi Aso ${ }^{d}$, Mark Barton ${ }^{a}$, Alessandro Bertolini ${ }^{a}$, \\ Valerio Boschi ${ }^{\mathrm{a}, \mathrm{c}}$, Dennis Coyne ${ }^{\mathrm{a}}$, Riccardo DeSalvo ${ }^{\mathrm{a}}$, Carlo Galli ${ }^{\mathrm{e}}$, Yumei Huang ${ }^{\mathrm{f}}$, \\ Alex Ivanov ${ }^{\mathrm{a}}$, Szabolcs Marka ${ }^{\mathrm{d}}$, David Ottaway ${ }^{\mathrm{b}}$, Virginio Sannibale ${ }^{\mathrm{a}}$, \\ Chiara Vanni ${ }^{\mathrm{e}}$, Hiroaki Yamamoto ${ }^{\mathrm{a}}$, Sanichiro Yoshida ${ }^{\mathrm{g}}$ \\ "LGO Laboratory, California Institute of Technology, MS 18-34, 1200 E. California Bivd., Pasadena, CA 91125, USA \\ ' LGO Laboratory, Massachusets institute of Technology, NW 22, 175 Albany Street, Cambridge, MA 02139, USA \\ ${ }^{\circ}$ Dipartimento di Fisica, Università di Pisa, Largo Bnino Ponteconvo 3, 56127 Pisa, Italy \\ ${ }^{d}$ Columbia University in the City of New York, 1009 Pupin Laboratory, New York, 10027 NY, USA \\ ' Galli \& Morelli s.r.l. Via Cristofani 558 loc. Acquacalda 55100 Lucca, italy \\ 'Department of Astronomy, Beijing Nonnal University, 100875 Beijing, PR China \\ ${ }^{8}$ Department of Chemistry and Physics, SLU 10878, Hammond, LA 70402, USA
}

\begin{abstract}
A B S T R A C T
New seismic pre-isolation systems will have to be included in the forthcoming gravitational wave interferometers of Advanced LIGO as part of the strategy to increase the sensitivity in the entire frequency range. In this perspective the LIGO Seismic Attenuation System (SAS) Group designed a new kind of seismic attenuation platforms for the Horizontal Access Module (HAM) and Basic Symmetric Chamber (BSC) vacuum chambers of the interferometers. HAM-SAS, a specific prototype for the HAM chambers, was constructed and installed in a test-bench laboratory. At the end of the commissioning time the prototype achieved many of the Advanced LIGO requirements about seismic isolation, stability and reliability. The lab experience also enabled us to finalize the design and indicated SAS as a solution worth considering for the seismic isolation of the advanced detectors.
\end{abstract}

\section{Introduction}

The LIGO ${ }^{1}$ interferometers accomplished their task in October 2007 after collecting 1 year of data at the design sensitivity (S5 science run). A major improvement of the sensitivity is expected from the upgrade of the interferometers to Advanced LGO [1] which should yield routine observation of gravitational waves. The Initial LIGO interferometers were mainly limited by seismic noise in the frequency band below $40 \mathrm{~Hz}$ [2], leaving a large margin of improvement before the fundamental floor of the gravity gradients noise at $-10 \mathrm{~Hz}$ is reached.

New seismic pre-isolation systems are required for the optics tables of both type of vacuum chambers of the interferometers, the Basic Symmetric Chamber (BSC) and the Horizontal Access Module (HAM). Whereas the first hosts the main test masses and the beam splitter mirrors, the second contains benches for the

\footnotetext{
" Corresponding author at: UGO Laboratory, California Institute of Technology. MS 18-34, 1200 E California Blvd., Pasadena, CA 91125, USA. Tel: +16263958507. E-mail address: stochino@ligo.caltech.edu (A. Stochino).

${ }^{1}$ The acromym stays for Laser Interferometric Gravitational wave Interferometer.
}

input/output optics of the interferometer (see Fig. 1 [3]). The Suspension Systems (SUS) adopted for Advanced LIGO to support the mirrors will either hang from the optics table, as in the BSC [4], or will be supported by it from below, as in the HAM [5]. A pre-isolation system of the in-vacuum optics tables will have to provide the first level of attenuation of the seismic noise needed to acquire and maintain the interferometer locking.

Over the last 10 years, the LIGO Seismic Attenuation System (SAS) Group has developed passive attenuation subsystems to isolate the suspensions from seismic noise. In view of Advanced UGO, the group has then designed complete seismic pre-isolation systems for both the HAM and the BSC chambers [6]. In this paper we present the results of the experimental tests carried on one of these prototypes, HAM-SAS, the one specifically designed for the HAM chamber.

\section{HAM-SAS system description}

HAM-SAS and BSC-SAS have been proposed searching for simpler and more reliable solutions for the Advanced LIGO isolation platforms than the HEPI-ISI active baseline [7]. In this 

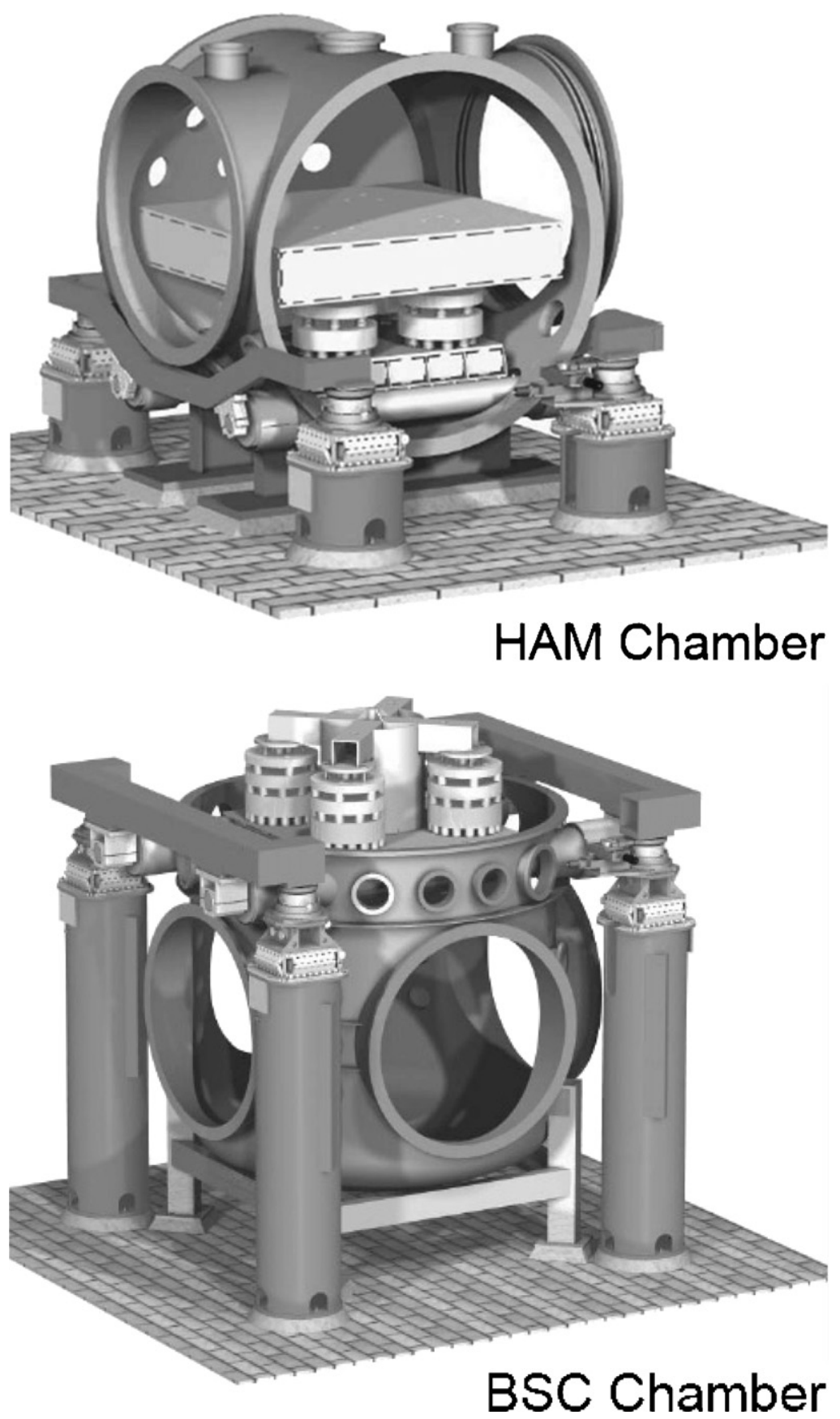

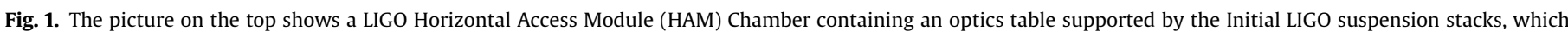

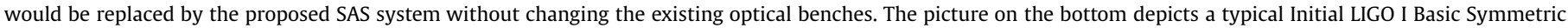
Chamber (BSC).

perspective, the systems can count on the established experience on the SAS passive attenuation technology after being largely tested and well proved at Virgo and TAMA [8,9].

Passive systems can offer several advantages in seismic isolation versus active systems. They are simpler, the attenuation being a product of the structural elasticity of the component materials. Their operation is not critically dependent on compound subsystems working together at the same time as in an active attenuation system. That makes passive system like SAS less prone to failure and thus, in principle, more able to provide the reliability required for high science-run duty cycles. Moreover, demanding minimal controls, with low cut-off frequencies and low gain, in principle they are less affected by noise re-injection problems. On the other hand, a passive system has its drawbacks as the critical dependence on the initial tuning and setup; the temperature effect on Young's modulus and thus on the springs elastic constant; the materials hysteresis at low frequencies. However, none of such limitations can be considered crucial and often it sufficient to take them into account in the system designing [10].

So, if SAS filters are mostly passive, their performances can be further improved with the assistance of active electronic controls. The passive and "soft" mechanical filters at the core of the system are justified by their typical features. As harmonic oscillators, these work as low-pass filters for the seismic excitations [11]. Considering ideal oscillators with one degree of freedom 


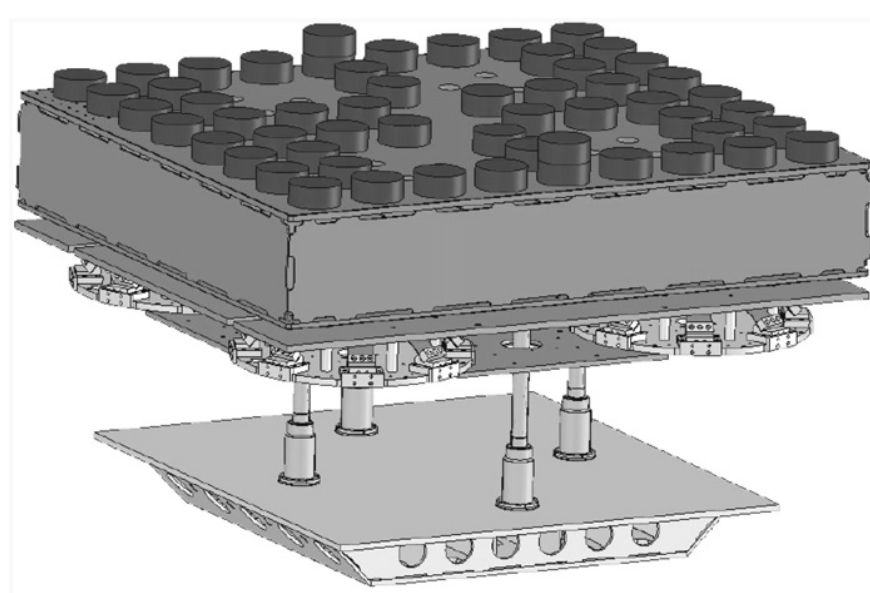

Each GAS filter (Fig. 3) is made of eight maraging arching steel blades [12], $3.44 \mathrm{~mm}$ thick, assembled onto a frame disk of $930 \mathrm{~mm}$ diameter [13]. The disks are mounted at the corners of the spring box and constrained between two plates. Stiffening side plates (not shown in Fig. 2) close the spring box and guarantee its rigidity. The filters simply support the same LIGO-I optics table on spherical tip pins in a quasi-kinematical configuration [14].

The spring box is supported by four IP hanging from the tips of their IPs' legs, connected by $30 \mathrm{~mm}$ long, $3 \mathrm{~mm}$ diameter, maraging steel wire joints. The IP legs are $448 \mathrm{~mm}$ long lightwall tubes, supported at the base by the main maraging steel flex joints, $50 \mathrm{~mm}$ long and $10.5 \mathrm{~mm}$ in diameter. Each leg weighs less than $300 \mathrm{~g}$ (including the stiffening over-thickness at the two ends) to minimize all attenuation limitations from Center of Percussion (COP) mismatch, and avoid internal modes at low frequencies. The flex joint at the base and the upper wire joint together form a double pendulum system supporting the table.

Below the main flex point, there is a larger diameter, $174 \mathrm{~mm}$ long, bell shaped, leg extension, made to support counterweights for the correction of the COP effect. In IPs this effect arises from the mismatch between the flex point and the rotation point caused at high frequency by the mass distribution in the leg [15]. The consequence is a notch in the transmissibility at about a few tens of Hertz followed by a plateau where the attenuation stops increasing [16]. An appropriate amount of counterweight below the flex point shifts the COP to overlap the flex point and improves the attenuation. It is SAS experience that well tuned IPs in such a configuration can have attenuation factors of more than $100 \mathrm{~dB}$ even with massive legs [17]. The light HAM legs already deliver $80 \mathrm{~dB}$ attenuation without COP compensation; therefore a COP compensation at the $10 \%$ level is sufficient to yield $100 \mathrm{~dB}$.

The COP effect occurs in GAS filters as well. The effect can be 1 compensated by means of a device, made of a light wand connected by flex joints between the blades' keystone and the frame disk to the blades' base. The wand carries a counterweight on the outer end and, by registering its position along the shaft, one can introduce the right amount of counteracting momentum of inertia and so reduce the COP effect and improve the filter's attenuation [18,19]. In HAM-SAS the GAS filters are designed to host up to four compensating wands (Fig. 4).

In the HAM chambers the optics table and payload are located above the rotation point of the table tilts (roughly at the level of the four keystones; see Fig. 5). This weight distribution introduces a destabilizing torque stronger than the recall torque from the GAS filters. To solve that problem a cross of four horizontal correction springs has been introduced below the center of tilt to add stiffness on the angular dofs [14] (see Fig. 5) without adding much to the stiffness in the vertical direction (see Ref. [14, Section 2.3] for an estimation). A vertical shaft is connected to the underside of the optics table, providing the central attachment point for the correction springs while, at the other end, these are connected to the four corners of the spring box. Since the springs are $4-5 \mathrm{~cm}$ long, $1 \mathrm{~m}$ long thin steel wires are used to cover the distance between the four corners and the shaft. This arrangement introduces a stabilizing torque proportional to the stiffness of the springs used and a vertical spring constant proportional to their tensioning. The required stabilizing torque can be obtained by choosing the adequate spring stiffness. These springs are minimally tensioned to remain in pull for the entire angular excursion of the table (few $\mathrm{mm}$ ) thus limiting their contribution to the vertical stiffness. The unwanted contribution to the vertical stiffness can be neutralized by adding a small additional radial compression to some of the blades of the four GAS filters. This compression has then to be adjusted according to the height of the payload center of mass to minimize the angular resonant frequencies.

\footnotetext{
${ }^{2}$ The transmissibility is the dynamical quantity in the frequency domain that measures the ratio between the displacement of the suspended mass and that of the suspension point.
} 


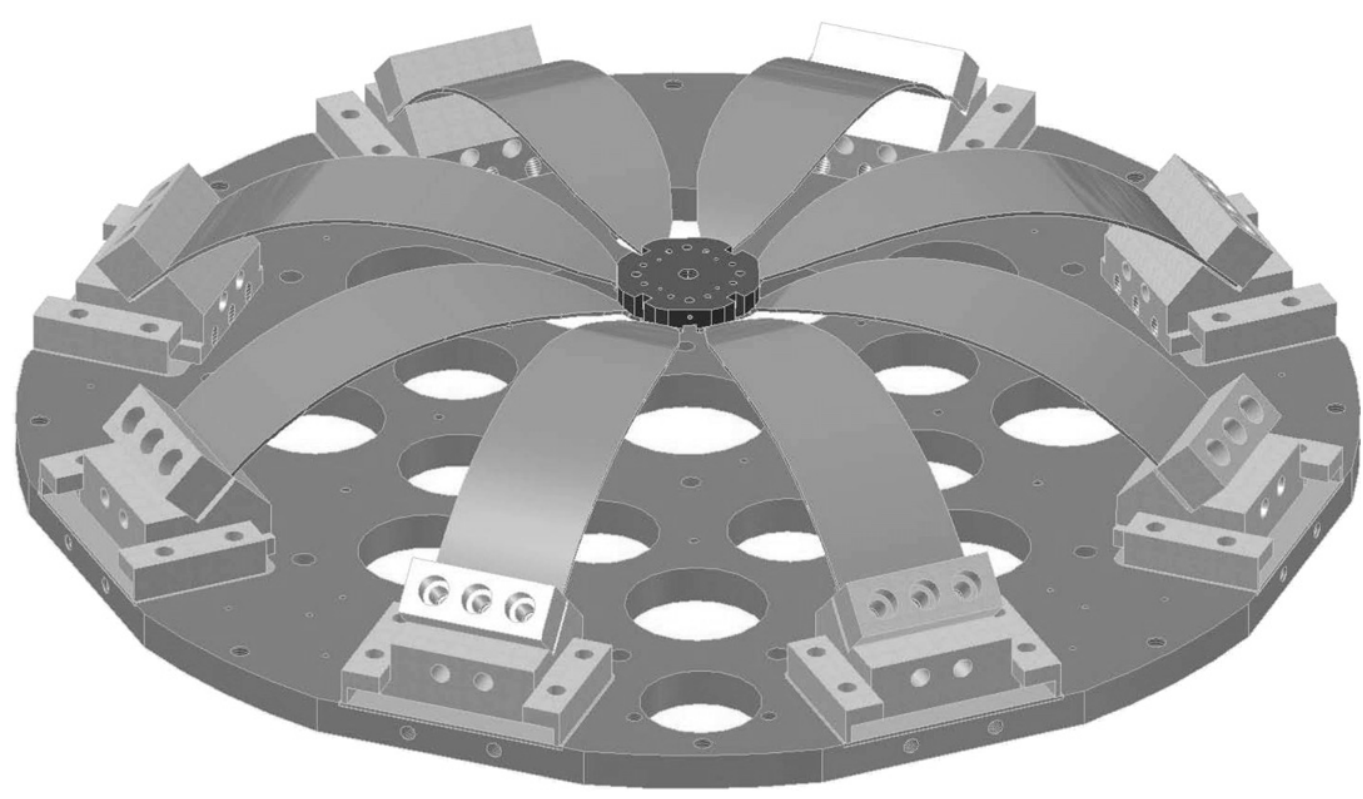

Fig. 3. HAM-SAS 8-blade Geometric Anti-Spring (GAS) filter.

\subsection{Sensors and actuators}

A consequence of the choice of soft suspensions is low actuator's power requirements. For static positioning the only control authority required is that necessary to compensate for tidal movements. For damping and feedback the actuators need only the control authority to counter the residual seismic power left over by the soft flexures. The power dissipated by an actuator is proportional to the square of the force it applies ${ }^{3}$ which, in turn, is proportional to the flexure elastic constant times the displacement. As the elastic constant is proportional to the square of the resonant frequency, $0.2 \mathrm{~Hz}$ or lower tuning results in a negligible power requirement-micro-watts for the seismic motion and few milli-watts for the tidal corrections-which can be easily provided by simple and highly linear non-contacting actuators [20] as well as low power coil drivers.

HAM-SAS has built-in LVDT (Linear Variable Differential Transformer) position sensors [21] for both vertical and horizontal stages. Four are located inside the GAS filters and, providing a signal proportional to the height of the filter keystones, measure the optics table's coordinates along the vertical dof. Four hang from the spring box, near the IP legs, and measure its position with respect to the base plate and thus the horizontal dof. These position sensors are used to provide resonance damping and control feedback to the co-located actuators. The performance measures obtained by lab measurements on the same LVDTs implemented in HAM-SAS are listed in Table 1.

The design of the system, but not the prototype, includes also the high sensitivity, UHV compatible, horizontal accelerometers developed by the SAS development group as inertial sensors. Mounted on the spring box, these are mostly immune to the tilts induced by the vertical suspension resonances and movements.

Eight stepper motors, four vertical and four horizontal, apply static offset forces and torques through soft springs to balance the system and precisely place it in the required position. The first four are mounted on the springs box and connected to the optics

\footnotetext{
${ }^{3}$ In an electromagnetic actuator the force depends on the coil current $I$ and the dissipated power as $I^{2} / R$.
}

table by coil springs, the other four are mounted on the base plate and connect to the spring box.

A prototype of the SAS design for the HAM chamber (HAM-SAS) commissioned by LIGO was built and assembled in Lucca (Italy) by Galli \& Morelli Srl. The system was then installed at MIT's LASTI lab to be tested in a LIGO HAM chamber. In this paper we discuss the results obtained from the laboratory tests.

\subsection{Design criteria}

The seismic pre-isolation systems have to be designed to absorb the Earth's crust seismic activity and the anthropogenic noise in order to maintain undisturbed interferometer operations and a duty cycle as close as possible to 100\%. The HAM- and BSCSAS design have been motivated by the need of a simple and effective solution to the issue of reliability of the Advanced LIGO isolation platform, in alternative to the HEPI plus ISI active baseline solution [7] that, containing hundreds of complex sensors and actuators, may lead to short time-between-failure time and long down times. Low frequency, passive pre-isolators proved very effective in isolating interferometers from external perturbations (human and small earthquakes) guaranteeing longer interferometer locks. ${ }^{4}$ The SAS design builds upon the positive experience on the passive attenuation technology tested and proved at Virgo and TAMA $[8,9,23]$ but uses similar, but improved techniques to provide the same reliability level.

Pre-isolation needs not necessarily to be separated from the rest of attenuation provided by the mirror suspensions. In Virgo, TAMA, LCGT, Aciga the pre-isolation is achieved within a compound system, using the same passive techniques throughout the entire chain. In LIGO, the choice of implementing optics benches to support both the main mirror suspensions and ancillary optics has introduced a physical separation between the two stages. The SAS design was adapted to provide the

\footnotetext{
${ }^{4}$ The super-attenuator system enabled Virgo to start its first scientific run with record length locks $[8,22]$.
} 


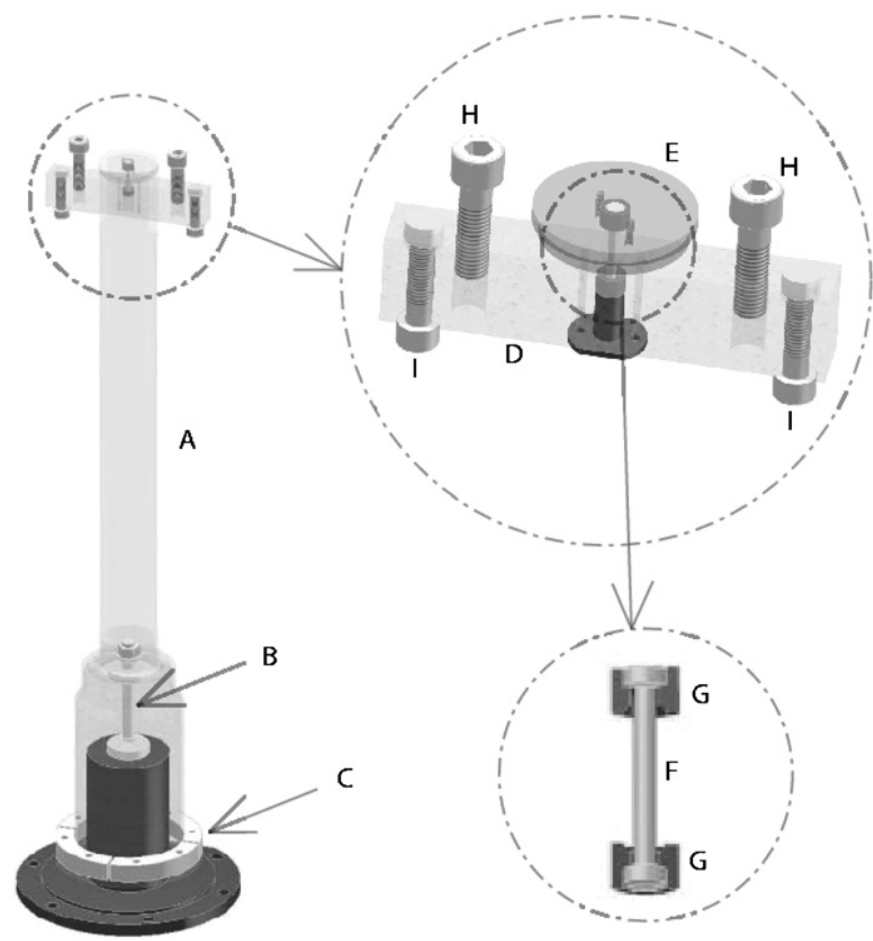

Fig. 4. The Inverted Pendulum leg $(\mathrm{A})$ is a light $50 \mathrm{~mm}$ diameter, aluminum tube. It connects to ground through a stiff cylindrical flex joint (B), provides all the restoring force of the Inverted Pendulum. Also rigidly connected to the bottom end of the leg is a light aluminum bell, which carries a circular counterweight mass (C) at its bottom rim. This counterweight brings the Center Of Percussion of the leg to coincide with the center of the flex joint (B). The top of the leg is sliced on a diameter for a width of $20 \mathrm{~mm}$ to allow the passage of the bridge (D) bolted to the spring box top plate. The top of the leg is closed by a circular cap (E). A thin wire flexure $(F)$, working in tension, extends from the cap to the bridge (D) providing a flexible connection to the spring box plate. This flexure provides negligible restoring force and acts like a free spherical joint. It is attached to the cap and to the bridge by means of two pairs of split cups $(G)$, which are inserted after the flexure is fed into its housings in the bridge and the disk, and hold the flexure in tension with pure compressive load (without shear stresses on the mating parts, which could generate stick and slip noise). The bridge is fastened to the spring box top plate (not shown) by means of the bolts $(\mathrm{H})$. The tuning bolts $(\mathrm{I})$, registered before the bolts $(\mathrm{H})$ are tightened, can micrometrically lift the spring box plate to equalize the load on the four legs. Load equalization is carefully determined by equalizing the $\sim 200 \mathrm{~Hz}$ resonance of the leg oscillating as a rigid body around the flexure (B) with the flexure (F) forming an " $\mathrm{S}$ ".

required seismic pre-isolation of the optical benches and to conform to this choice.

The SAS was designed according to the following reliability criteria:

- Instruments' reliability: Only passive sensors and actuators, made of polyimide-coated wire wound on Peek spools, are used inside the vacuum tanks. The inductive signals are carried outside the vacuum enclosure with twisted pairs requiring no in-vacuum active circuitry. These instruments, except for physical impact and connector failures, are essentially free of failure modes. The elimination of in-vacuum active electronics also adds up to the overall system reliability.

- Redundancy: To provide sufficient reliability, no single sensor or actuator should be critical for system operation, therefore four sensor/actuator units were foreseen for each group of three dofs. Failure of a unit results in marginally diminished performance, not requiring immediate substitution to maintain the interferometer observational reliability.
- Vacuum facility risks: Only completely UHV compatible instruments are used.

(1) No enclosed gas volume is allowed, to eliminate the possibility of potential leaks hardly localizable, for cleanliness protection of the mirrors and UHV infrastructure.

(2) The assembled and pre-tuned structure is designed for an additional final vacuum preparation bake at $120^{\circ} \mathrm{C}$, to eliminate the risk of vacuum pollutants accidentally deposited on the parts during assembly.

(3) Safe actuation power requirements: Low power coils, tolerating indefinite exposure to a railed power supply without out-gassing or damage, are employed. The soft spring support makes these weak actuators sufficient to shift or tilt the optics bench as necessary (large optical table excursions are obtained by means of static stepper motors controlled auxiliary springs occasionally powered). If any active control were needed, because of the passive attenuation design, the weak actuator authority would be largely sufficient to actively cancel the residual seismic motion after the mechanical filters but, still, it would not have enough power to shake the table beyond the level of normal ground seismic activity. Their authority is also sufficient to counter the internal back-reaction forces (e.g. forces generated by the actuation of the supported multiple pendulum).

(4) No steady power dissipation (no more than a few mW) allowed inside UHV to minimize thermal gradients. Auxiliary springs attached to stepper motor slides are used to determine the static equilibrium position of the optics table and eliminate standing correction forces, so that no significant heat is generated in the voice coil actuators under vacuum. As there are no steady forces, in case of power failure and/or sudden actuator shutdown the optics table remains close to its equilibrium position without vibrations imposed by sudden loss of static forces to the payload.

- Earthquake damage safety: A $10 \mathrm{~mm}$ range of the low frequency isolation in all dofs was designed to protect the optics benches from large seismic excursions (twice as large as Olympia's earthquake; see Ref. [24]).

- Lock loss from distant earthquakes strategy: SAS is designed according to Virgo's successful control strategy to maintain the interferometer's lock under such seismic events [25].

- Minimized complexity and maintenance risks: The SAS design satisfies the Advanced LIGO pre-attenuator requirements in a single, mostly passive, stage versus the two or three active stages of the baseline design. HAM-SAS mechanics is designed to make the vertical and the horizontal groups of dofs as independent as possible. No maintenance and re-tuning are required from a passive system because the system is baked under full stress after assembly. Even though the flexures are not subjected to high stresses, this baking prevents any creep and any possible creep-caused re-tuning in the experiment lifetime [26]. Minimized complexity brings minimized construction, implementation, commissioning and maintenance costs, both in financial and personnel terms.

The design also includes the following features that enhance the system operational flexibility.

- The SAS GAS springs are designed with a variable number of blades and dimensioning, to match the wanted payload. So do the IP flex joint to tune the stiffness needed for the payload. This reduces the necessity of large ballast masses on the less loaded optical benches. 


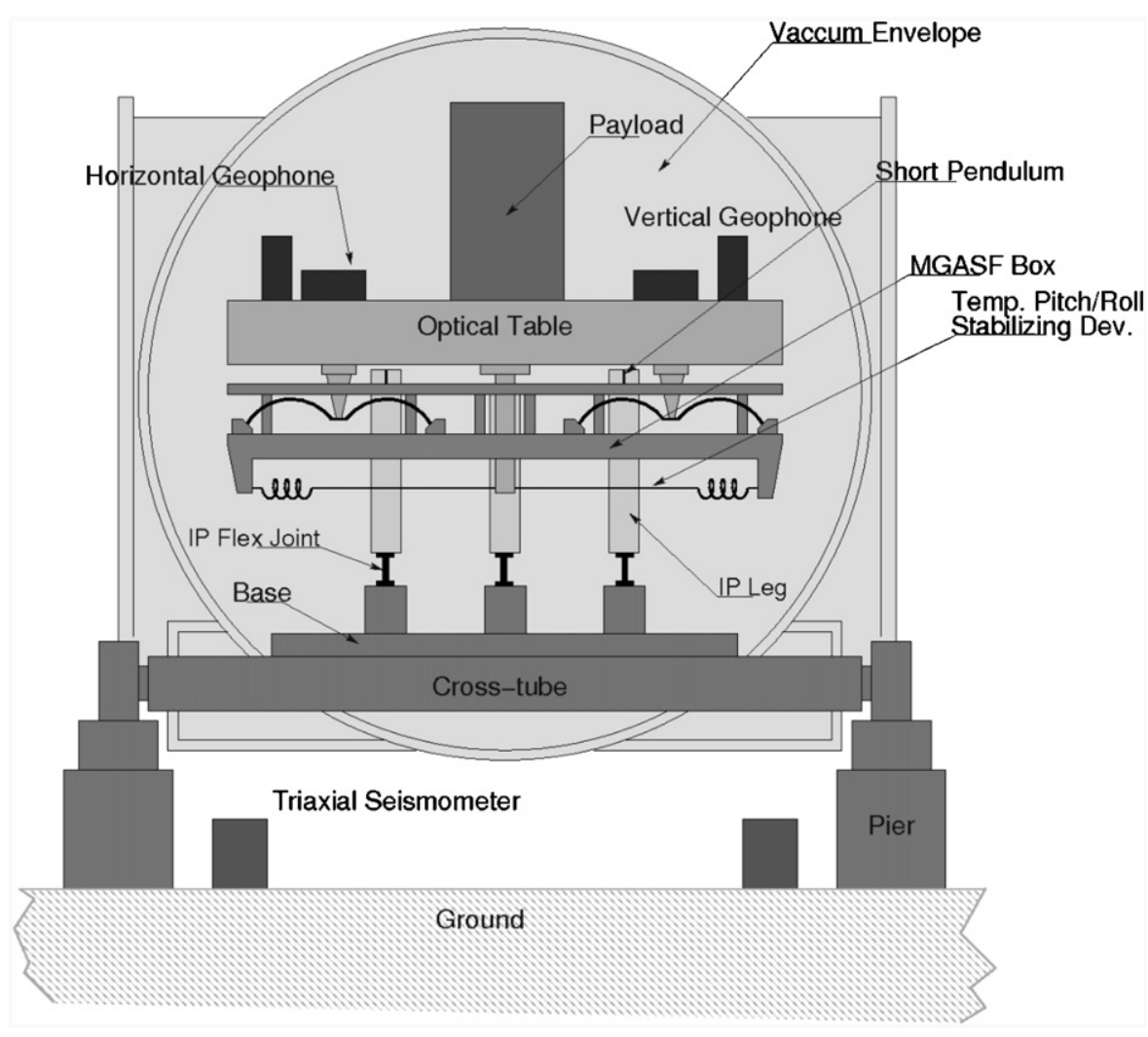

Fig. 5. Scheme of the tilt stabilization springs added between the optical plate and the spring box.

Table 1

LVDT performances, measured in laboratory bench tests.

\begin{tabular}{lll}
\hline & Horizontal LVDT & Vertical LVDT \\
\hline Deviation from linearity & $0.88 \%$ & $0.26 \%$ \\
Sensitivity & $6.49 \mathrm{~V} / \mathrm{mm}$ & $7.85 \mathrm{~V} / \mathrm{mm}$ \\
Range & $20 \mathrm{~mm}$ & $20 \mathrm{~mm}$ \\
Displacement noise & $2 \mathrm{~nm} / \sqrt{\mathrm{Hz}}$ at $10 \mathrm{~Hz}$ & $2 \mathrm{~nm} / \sqrt{\mathrm{Hz}}$ at $10 \mathrm{~Hz}$ \\
\hline
\end{tabular}

The deviation form linearity is the local variation of the position-to-voltage coefficient across the $20 \mathrm{~mm}$ range. The high sensitivity is conserved throughout the range.

- It is sufficient to align the center of mass only in the horizontal plane with no need of ballast mass for vertical balancing around the support/pivot point making the system lighter and easier to handle.

- UHV compatible tooling was designed to allow fast, safe and clean installation of the HAM-SAS unit and of the optics bench within a working day. The HAM optics bench installs independently, simply sliding over the platform allowing the installation of pre-populated optics benches.

- The low frequency tune of the SAS strongly decouples its payload and intermediate rigid platforms from the ground, thus making those platforms ideal for active controls with minimal control authority. Placements for optional performance-boosting accelerometers are foreseen. The horizontal accelerometer positioning would be on the spring box, ahead of the tilting stage, to mitigate the cradle effect plaguing six dofs active. The vertical accelerometers would be placed in the optical table. The accelerometers would be custom design units, fully UHV compatible [27] with low frequency performance exceeding the best available seismometers.
- The whole structure and the design parts tolerances make SAS compatible with high-temperature bake-out-induced warping. All individual parts, including aluminum parts and coils, are designed to undergo a $200{ }^{\circ} \mathrm{C}$ bake-out. Registering is foreseen for all sensitive points to compensate for residual deformations after assembly.

- Coil-based, large-stroke position sensors are used. They do not require position re-tuning even for relative large changes of optics table positioning.

\section{HAM-SAS control}

Rigid platforms in free motion on determined modes form an ideal plant for active controls with global interferometer feedback signals, on-ground seismometer feed-forward or, if sufficient sensitivity is available, on-board accelerometers.

\subsection{Diagonalization}

The number of the sensor/actuator units (four for three dofs) and their positioning are chosen to match the "rectangular" symmetry of the vacuum chamber, to provide redundancy of sensing and actuation, and to maximize their control efficiency. The coordinates of the optics table are measured with respect to an orthogonal frame referred to the lab. Let $\mathbf{u}=\left(h_{1}, h_{2}, h_{3} h_{4}\right.$, $v_{1}, v_{2}, v_{3}, v_{4}$ ) be the coordinates from each horizontal and vertical LVDT, and $\mathbf{x}=\left(x, y, z, \theta_{x}, \theta_{y}, \theta_{z}\right)$ the coordinates of the geometrical center of the table respect to a Cartesian frame referred to the lab. We have that $\mathbf{x}=U \mathbf{u}$, where $U$ is a $6 \times 8$ matrix in the coordinate space that changes the basis. In the same way, for the actuators we can define the vector $\mathbf{v}=\left(f_{h 1}, f_{h 2}, f_{h 3}, f_{h 4}, f_{v 1}, f_{v 2}, f_{v 3}, f_{v 4}\right)$, with the forces applied by each coil, and the vector $\mathbf{q}=\left(q_{x}, q_{y}, q_{z}\right.$, $q_{\theta x}, q_{\theta y}, q_{\theta z}$ ), with the total forces and torques applied to the table 


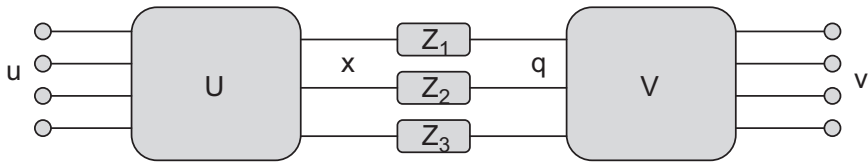

Fig. 6. Diagonalization scheme. u represents the signals from the four real position sensors for either the horizontal or the vertical degrees of freedom. These are converted into coordinates $\mathbf{x}$ of virtual sensors by the sensing matrix $\mathbf{S}$. Compensation filters $\mathbf{Z}$ provide the forces $\mathbf{q}$ to the virtual actuators oriented as the virtual sensors $\mathbf{x}$. The virtual forces are projected into real forces $\mathbf{v}$ on actuator coils by the driving matrix $\mathbf{D}$. If the sensing matrix $\mathbf{S}$ and the driving matrix $\mathbf{D}$ are multiplied to the $\mathbf{U}$ and $\mathbf{V}$ matrices, respectively, then the filters $\mathbf{Z}$ work independently on the natural modes of the system.

along the Cartesian coordinates. It is $\mathbf{v}=V \mathbf{q}$, where $V$ is the $6 \times 8$ matrix in the space of forces changing the basis. Positions and forces are related by the transfer function of the system which has the form of a $6 \times 6$ matrix $H$, so that $\mathbf{q}=H \mathbf{x}$. The optics table, as a rigid body, has six dofs and, in first approximation, along each of these, it can be represented by a harmonic oscillator. Six normal modes exist for the overall motion of the system [28] (Fig. 6).

In general, $H$ is not diagonal, that is each actuator excites all six modes. However, $H$ can become diagonal if basis oriented as the normal modes are chosen for $\mathbf{x}$ and $\mathbf{q}$. In the new basis $\mathbf{x}^{\prime}=S \mathbf{x}$, $\mathbf{q}^{\prime}=D^{-1} \mathbf{q}$, where $S$ and $D$ are usually referred to as sensing and driving matrices, respectively, and $\mathbf{x}^{\prime}=H^{\prime} \mathbf{q}^{\prime}$, where the transfer function $H^{\prime}=D^{-1} H S^{-1}$ is now diagonal. For this reason, the process is called diagonalization.

\subsection{Measuring the sensing and the driving matrices}

The way we measured $H^{\prime}$ can be summarized as follows (see Ref. [14] for the details). $S^{-1}$ is measured from the relative amplitudes between the sensors' readout in correspondence with each mode. For the driving matrix, one may either directly assume it to be equal to the transpose of $S$, relying on the fact that the coil actuators are coaxial with the LVDT, or obtain it from the measurements of the ratios $(N)_{i j}=\left(q_{i} / x_{j}^{\prime}\right)_{\omega=\Omega}$ at a fixed excitation frequency $\Omega .^{5}$ Since $\mathbf{q}=D H^{\prime} \mathbf{x} \equiv N \mathbf{x}, N \propto D$ because $H^{\prime}$ is diagonal. Then $H^{\prime}$ is obtained from the ratios $\left(q_{i}^{\prime} / x_{j}^{\prime}\right)$.

In HAM-SAS the mechanics naturally separates the horizontal and the vertical dofs. It is therefore useful to separate the modes into two groups (or stages), the vertical and the horizontal, and to consider them as independent from each other. This simplification breaks the problem of the diagonalization into two formally identical ones. $H^{\prime}$ can be separated in four blocks, with non-null elements only in the two blocks on the diagonal:

$H^{\prime}=\left[\begin{array}{ll}{[h]} & {[0]} \\ {[0]} & {[v]}\end{array}\right]$.

If the system behaved completely linearly, we would expect the sensing and the driving matrices to remain constant for all the frequencies. However, non-linearities change the system response and an optimal diagonalization may be frequency dependent for a fully optimized diagonalization (Fig. 7).

\section{Physical plants transfer functions}

After the diagonalization, the force-to-position transfer function of the system was measured along all the modal coordinates (see Fig. 8).

We found the curves matching harmonic oscillator functions with resonant frequencies from 30 to $80 \mathrm{mHz}$ and $Q \backsim 5$ along the

\footnotetext{
${ }^{5} \Omega$ has to be much lower than any resonant frequency of the modes.
}
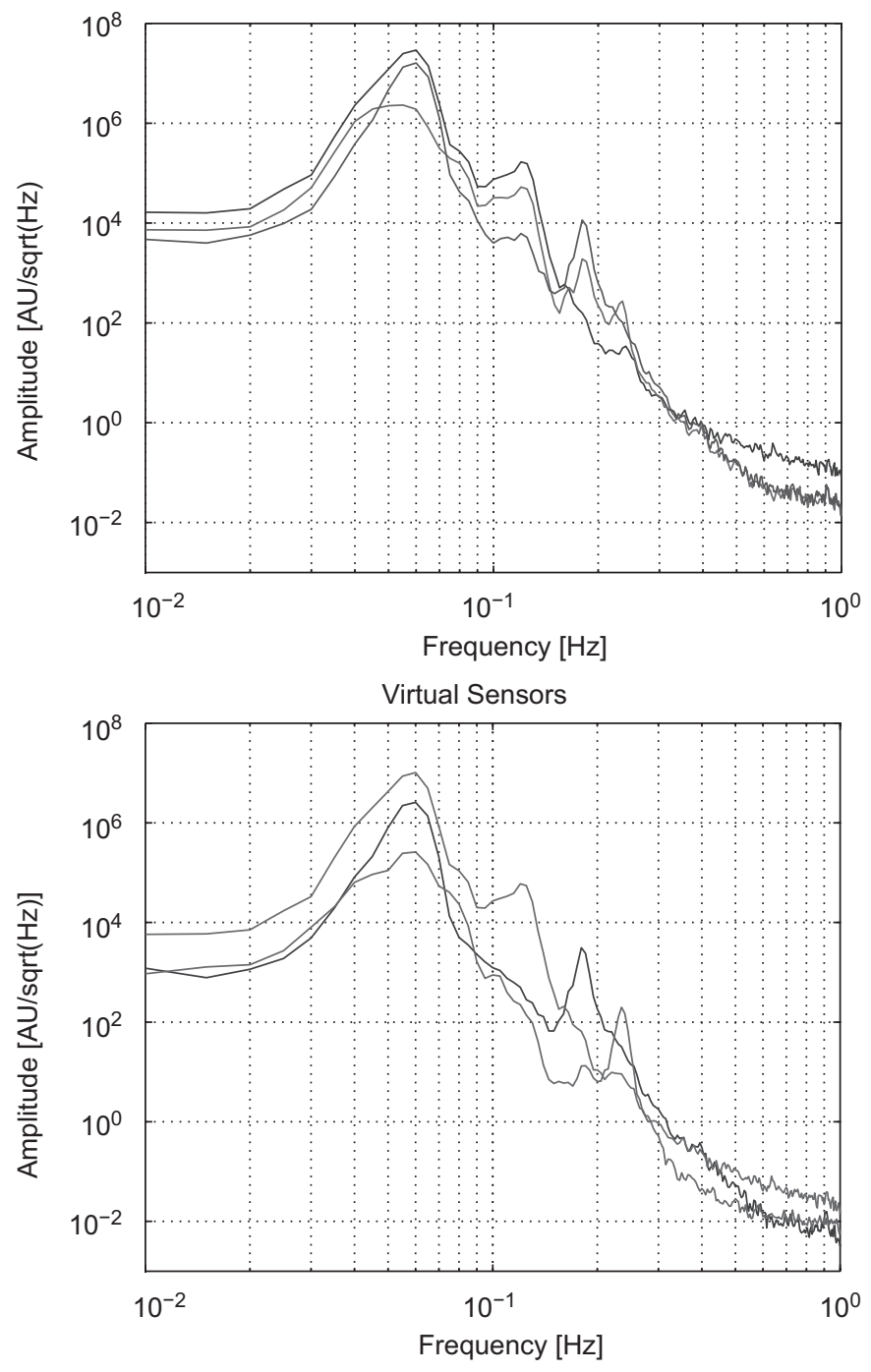

Fig. 7. Vertical diagonalization. The plots show the power spectrum amplitudes of the vertical coordinate signals in the non-diagonalized case (upper plot) compared to the corresponding diagonalized sensor signals (lower plot). In the nondiagonalized case all three sensors are equally sensitive to the first two resonances while the third peak is already partially decoupled. After diagonalization each sensor is only sensitive to a single resonant mode (single peak) with at least a factor of 10 in rejection of the unwanted modes. The large resonant peak below $0.1 \mathrm{~Hz}$, visible in all sensors even after diagonalization, is due to yet un-damped horizontal motion coupling into the tilts of the overhanging payload.

horizontal dof, and from 150 to $250 \mathrm{mHz}$ and $Q \backsim 10$ along the vertical. These frequencies correspond to the last mechanical tuning before the chamber was closed and the vacuum pumped. Lower frequency values could have been achieved by a finer tuning of the IP load mass, the compression of the GAS blades and an optimization of the coil springs stabilizing the tilts.

It is in the philosophy of passive isolation to aim to the lowest possible resonant frequencies. The reasons are several:

- the attenuation, proportional to $\omega^{-2}$, starts earlier and then, for a given frequency, is larger;

- the actuation force necessary to induce a given displacement in the suspended mass is smaller (proportional to the square of the resonant frequencies);

- the quality factor of each resonance scales with the square of its resonant frequency and requires less or no damping at lower frequencies $[11,14,16]$. 
$X$ Mode Frequency $\mathrm{f}_{\mathrm{x}}=37 \mathrm{mHz}$

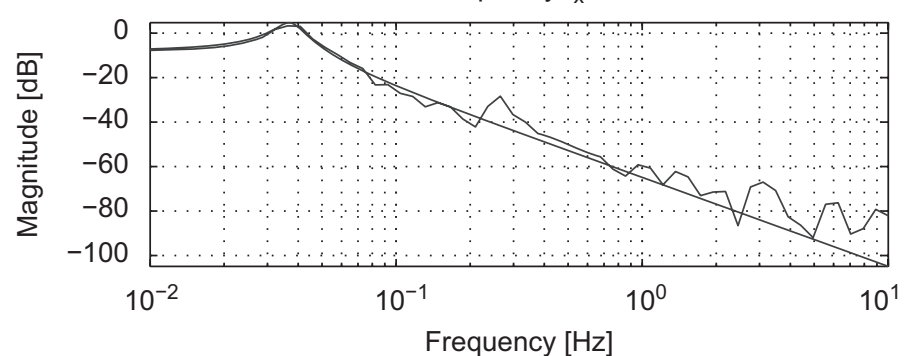

$Y$ Mode Frequency $f_{y}=59 \mathrm{mHz}$

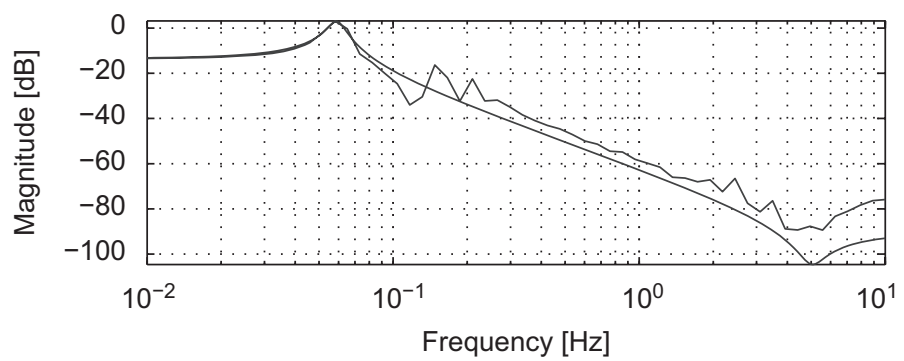

$Z$ Mode Frequency $f_{z}=148 \mathrm{mHz}$

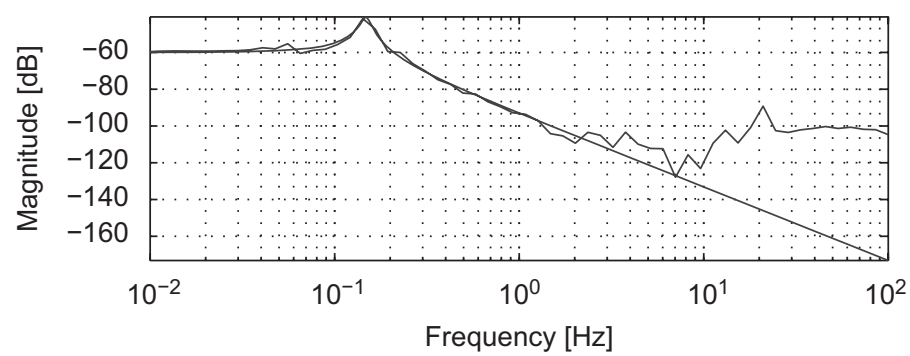

$\theta_{\mathrm{x}}$ Mode Frequency $\mathrm{f}_{\theta \mathrm{x}}=200 \mathrm{mHz}$

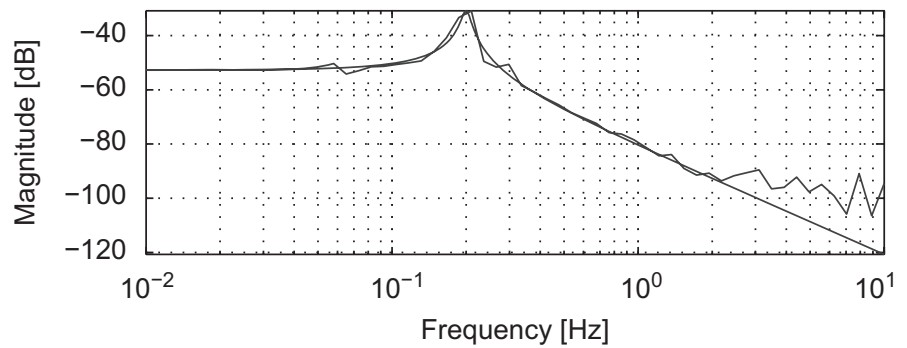

$\theta_{\mathrm{y}}$ Mode Frequency $\mathrm{f}_{\theta \mathrm{y}}=245 \mathrm{mHz}$
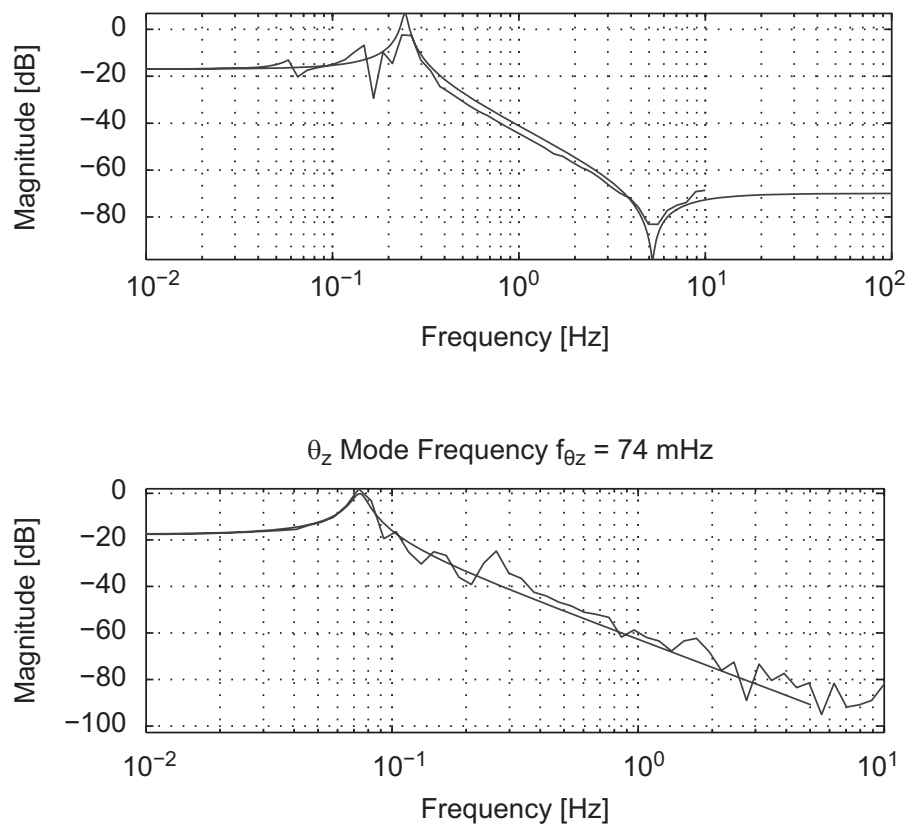

Fig. 8. Modal transfer functions of the diagonalized six degrees of freedom. The saturation in $Z$ above $10 \mathrm{~Hz}$ is probably connected with the lack of COP compensators.

Setting IP and GAS filters to excessively low frequencies may lead to instability. Perturbations, due to e.g. vacuum chamber distortion during the pump-down can slightly change the balance of the system. For prudence in the first pump-down we decided to leave some safety margin. To compensate we implemented an aggressive control strategy to electronically lower the resonant frequency (EMAS). As doing this is prone to control noise reinjection, the set configuration was far from optimal.

\section{Platform control}

In the designed system, where low natural frequencies are sought, the role of the control system is minimal, mainly intended to set the mechanical system in its optimal working point and in part also to improve the attenuation performance. The controls can be "passive", based on the LVDTs, which work as relative position sensors, or "active", based on the accelerometer's inertial response, or a combination of both.

The scheme of the passive controls for a single dof is shown in Fig. 9. Here $x_{0}$ represents the reference position (i.e. the ground for the IPs, the spring box for the GAS filters), whereas $x$ is the corresponding system's coordinate (i.e. the horizontal displacement of the IPs' end or the position of the GAS blades' keystone). $G$ is the transfer function of the mechanical system along the $x$ dof, the so-called physical plant response, and $C$ is the compensator filter. The closed loop transfer function

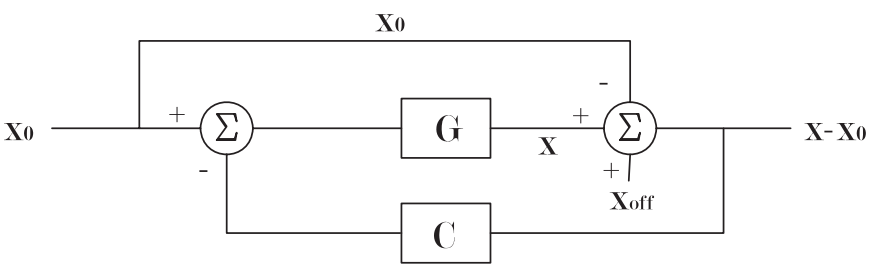

Fig. 9. Control topology with relative positions sensors as the LVDTs.

of the system is

$x(\omega)=\frac{G(\omega)(1+C(\omega))}{1+G(\omega) C(\omega)} x_{0}(\omega)+\frac{G(\omega) C(\omega)}{1+G(\omega) C(\omega)} x_{o f f}(\omega)$.

In the active topology, where there is no subtraction of the reference position, the closed loop transfer function would not contain the factor $(1+C(\omega))$ at the numerator.

The system prototype had no built-in accelerometers on the spring box so that the controls that we tested were only passive. We performed some tests to actively control the platform using witness L4c geophones as inertial sensors. Although we did not thoroughly explore this strategy, we closed the loops in all six dofs proving the possibility to implement inertial controls [29].

In the LASTI tests, which reproduced the conditions of the sites, the controls were implemented by the LIGO Control and Data Systems (CDS) [30,31]. All the input and output signals were provided by either the ADC or the DAC and the controls were 
handled by an EPICS digital computer interface. Here, the loop filters were designed in the frequency domain and automatically translated into the time domain.

\subsection{Damping control}

The quality factors of the resonances in the dofs can be reduced by damping feedback forces, that is by a derivative filter $C(\omega) \backsim i \gamma \omega$.

\subsection{Static position control}

It is important that the optics table can be brought to, and maintained at, the position required by the interferometer operation. This automatically keeps IPs and GAS filters at their optimal working point if the optical payload is properly positioned. The control loop to maintain the table at an assigned point $x_{o f f}$ is implemented by an integration filter $C(\omega)=\alpha / \omega$, in which $\alpha$ sets the gain factor, effectively its integration time constant. Assuming $G(\omega)$ is a pure harmonic oscillator transfer function-as we expect it to be if the considered dof is sufficiently decoupled from the others-then a necessary criterion for the loop stability imposes that the integration time $\tau=1 / \alpha$ be chosen compatibly to the resonant frequency of the system $\omega_{0}$, so that $\tau \gg 1 / \omega_{0}$.

\subsection{Electromagnetic anti-spring}

The resonant frequency of a harmonic oscillator can be lowered by introducing an Electromagnetic Anti-Spring (EMAS) in parallel to it [32]. The system is based on a control loop in which the feedback signal is frequency-independent and proportional to the output. The resonant frequency can be lowered or increased by changing the sign of the feedback. Referencing to Eq. (1), $C(\omega)=-k, k$ being a constant, so that the feedback is positive. Assuming $G(\omega)=\omega_{0}^{2} /\left(\omega_{0}^{2}-\omega^{2}\right)$, according to Eq. (1), the closed loop transfer function is

$G_{c l}(\omega)=\frac{\omega_{0}^{2}(1-k)}{\omega_{0}^{2}(1-k)-\omega^{2}}$

which is the transfer function of an oscillator with an effective resonant frequency reduced by a factor $\sqrt{1-k}$. Care must be used to limit the feedback signals to avoid making the system unstable $(k>1)$.

With an approximate diagonalization, applying anti-springs to a mode affects the others and "drags" their resonant frequencies. When attempting to tune all six modal oscillators to low frequency, the shift of the EMAS in a dof can destabilize another one. A preliminary test was performed on the vertical dof. A rough diagonalization of sensors and actuators was made assuming the modes of the system were pure vertical translation ( $z$ mode) and two rotations along the Cartesian axis of the lab $\left(\theta_{x}\right.$ and $\theta_{y}$ angular modes). ${ }^{6}$

In order not to destabilize the system, we left the two angular modes $^{7}$ in their mechanical tuning at 130 and $195 \mathrm{mHz}$. Static position control loops were applied on the other five dofs.

We applied the EMAS to drag the vertical resonance down from its mechanical frequency at about $150-10 \mathrm{mHz}$. We traced in

\footnotetext{
${ }^{6}$ We used the averaged sum of the four vertical sensors to generate the vertical feedback signal and sent the same signal in parallel to the four vertical actuators to generate the vertical actuation.

7 The actual modes of the system turned out to be not very far from these. When we say to vertical or angular modes in such a Cartesian diagonalization, we mean the mode with the largest vertical projection or one of the angular projections, respectively.
}

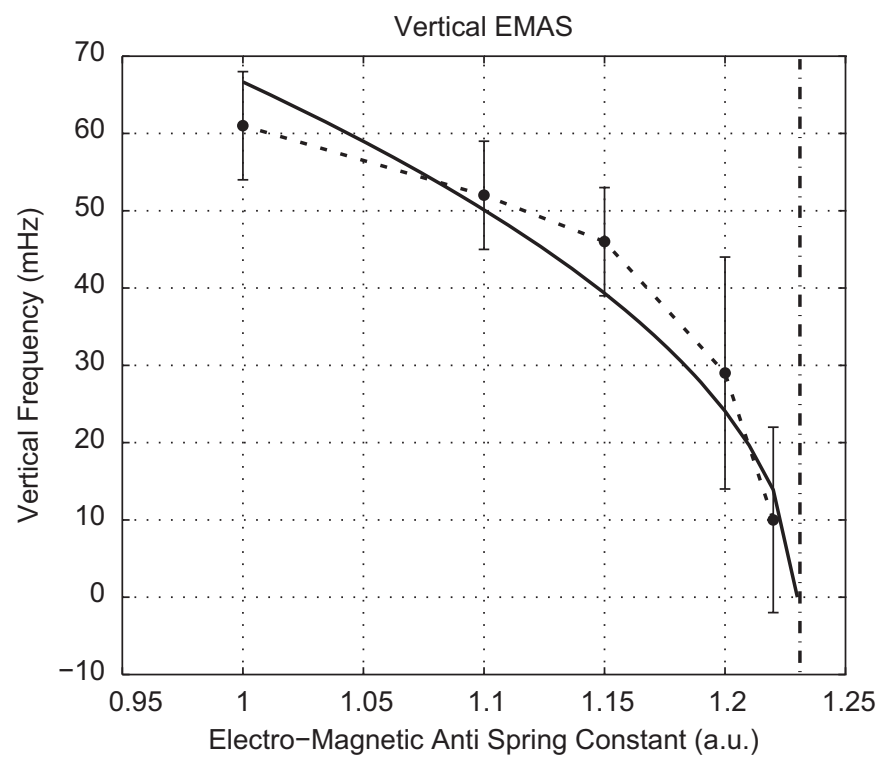

Fig. 10. Vertical resonance frequency as a function of EMAS gain. The error bars represent the measured width of each resonance peak. The solid line is a square root function fit to the data. The vertical dashed line is the critical EMAS gain corresponding to zero recall force.

Fig. 10 the evolution of the vertical resonance as a function of EMAS gain.

To close the EMAS loops on all the dofs at the same time would demand a fine diagonalization of the system. If this condition does not hold, as in the mechanical tuning conditions of the present tests, because of the high EMAS gain necessary to drive the frequencies towards zero, the coils drivers can saturate and even mild seismic excitation may trigger instabilities. We therefore settled for moderate EMAS gains in all three vertical dofs, without trying to lower much the resonant frequencies. This kind of control demonstrated to be effective and stable.

The same method can be independently applied in the horizontal dof. Low-pass filters could be added in more advanced controls to reduce the EMAS authority at high frequency and prevent noise re-injection from the ground-referred position sensors.

\section{Attenuation}

The power spectrum amplitude of the residual motion of the optics table and the transmissibilities between ground and the table were measured to characterize the seismic attenuation of the system. The first is measured by three vertical and three horizontal Mark Products L4-C geophones mounted at the periphery of the optics table, roughly in a triangular configuration. The transmissibility is evaluated between three Guralp CMG-T40 seismometers on ground and the six geophones Fig. 11.

The geophones are inertial sensors of relative velocity. Their sensitivity to the displacement is proportional to $\omega^{3}$ and it is significantly limited at low frequencies, below the internal mass' resonance (about $0.7 \mathrm{~Hz}$ ). In a more subtle way, because of the Principle of Equivalence, they are affected at low frequency by tiltto-horizontal coupling, which prevents them from distinguishing the horizontal accelerations from the angular $\left(\theta_{x}, \theta_{y}\right)$. Furthermore, the signal coming out of the L4C electronic driver boards includes a DC component requiring a filter that prevents any significant measurement below $100 \mathrm{mHz}$. These considerations about the sensors' limitations allow accurate performance analysis only for frequencies above $100 \mathrm{mHz}$. 

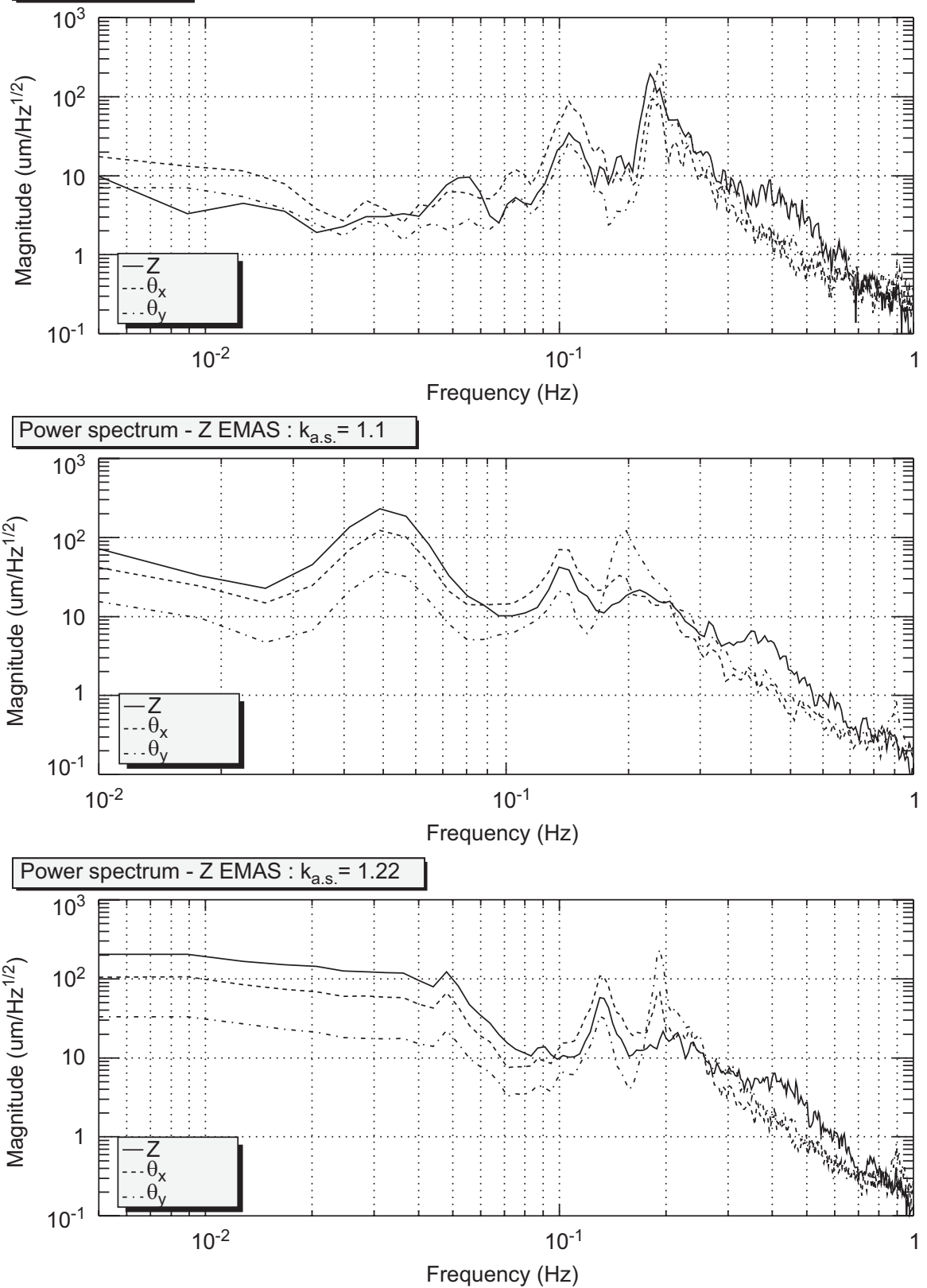

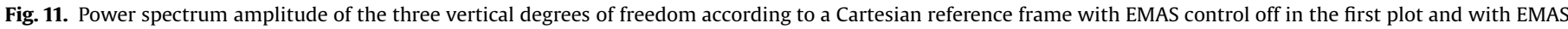

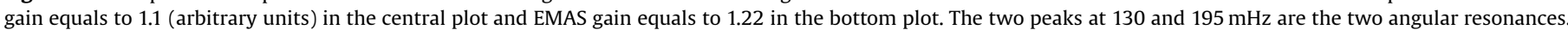

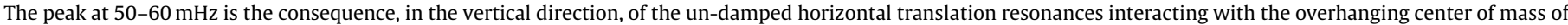

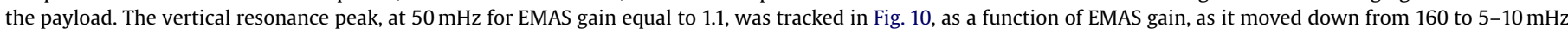

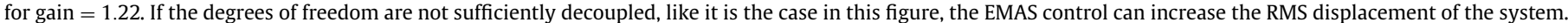
The measurement shown in here were meant only to test the efficacy of the EMAS in lowering the resonance peaks.

The most important indicator to evaluate the quality of the measurements of transmissibility is the coherence among the sensors. In particular, low values of coherence between the Guralps and the geophones affected the measurements of transmissibility above a few Hertz, for the horizontal dof, and, above about $20 \mathrm{~Hz}$, for the vertical. Also, the poor coherence among the Guralps themselves prevented the measurement of the ground angular seismic noise, which remains still substantially unknown.

Fig. 13 shows the power spectra of the table's residual motion along the laboratory frame, compared with the correspondent seismic ground spectra. The measurements were made with the DC and damping controls on. The dashed lines represent the LIGO requirements for the HAM chambers. The plot shows that the measurement meets the requirements in most of the frequency range with exceptions where some more observations are necessary. For instance, a residual motion exceeding the ground's seismic displacement is present at $100 \mathrm{mHz}$ for one of the horizontal directions. The horizontal readout of the geophones does not provide a precise measurement of the importance of the effect since it unavoidably contains contributions due to coupling with the angular dofs. ${ }^{8}$ Nonetheless, it is reasonable to identify

\footnotetext{
${ }^{8}$ The limitation, known as tilt-to-horizontal coupling, unavoidably affects that kind of inertial sensors, especially at low frequency $[14,33]$.
} 
the source of the observed exceeding power on imperfect controls designed over a not fine enough diagonalization of the physical plant matrix. Supposedly some of the closed-loop transfer functions had not a very large margin of stability with respect to a residual mode left in by the diagonalization. Such notoptimized controls also justifies the not entirely damped motions between 0.2 and $1 \mathrm{~Hz}$. Also, more or less distinguishable peaks appear at about $200 \mathrm{mHz}$ in all dofs in coincidence with the vertical modes resonances. Those were probably due to power sneaked through an imperfect diagonalization. Finally, the marked peak at about $20 \mathrm{~Hz}$ is a spurious resonance that appeared after the installation of the tilt stabilizing springs' steel wires. The resonance at $20 \mathrm{~Hz}$ can be mechanically damped by tying a cut rubber O-ring halfway along the wires, as successfully demonstrated in laboratory test at Caltech. The cut O-ring forms a " $\mathrm{C}$ " with an opening and closing frequency much lower than all wire violin modes. The wire violin modes force the " $\mathrm{C}$ " to open and close and get efficiently damped by the rubber viscosity. A damping factor of 30-100 was observed in a lab test, getting the wire close to critical damping. However, neither of these points is due to a structural problem, nor represents a serious issue that a commissioning cannot solve. It is reasonable to expect that with proper tuning on the system the power spectra would match the requirements in the entire frequency range.

Most of these problems of controls and imperfect diagonalization could be avoided if the whole system had been subjected to a finer mechanical tuning. The GAS filters have to be tuned to lower frequencies to compensate for the vertical stiffness added by the tilt stabilizing springs, and more tuning mass on the spring box would lower the horizontal frequencies of the IPs below $30 \mathrm{mHz}$ in the horizontal dof. Lower frequency tune would also move the mechanical filters further along their $1 / f^{2}$ attenuation curve, thus correspondingly improving the transmissibility curves. More natural damping of the vertical resonances would occur with the lower frequency tune as in the design, thus significantly reducing the need of active control damping, according to SAS passive strategy.

SAS is designed as a primarily passive attenuation unit, with minimally active controls, mainly for static positioning and suspension resonance suppression. Its design resembles that of a large accelerometer's inertial mass. Soft flexures support the payload, while isolating it from the external perturbations. Like in seismometers, the flexures are designed to reach the lowest practical resonance frequencies. The isolation of seismic noise is limited by the flexure material's characteristics (elasticity limit,

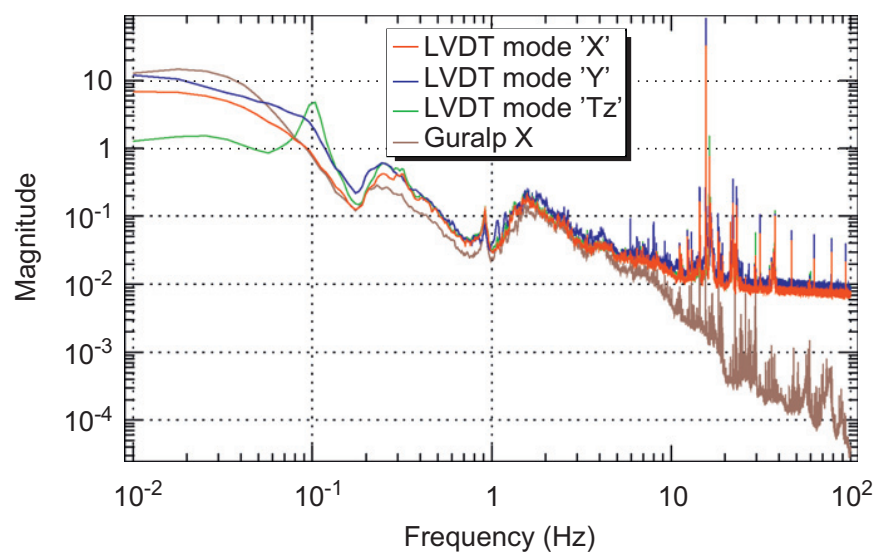

Fig. 12. The plot shows how the power spectrum amplitudes of the LVDTs and that of the Guralp seismometers match below a cut-off frequency (about $2 \mathrm{~Hz}$ ). That demonstrates how the system with its LVDTs behaves as a seismometers for sufficiently low frequencies. dissipation characteristics and hysteresis), therefore the SAS response to the seismic noise ideally approaches that of a very sensitive accelerometer (see Figs. 12 and 13).

The transmissibilities in Fig. 14 show that the attenuation reaches about $70 \mathrm{~dB}$ in the horizontal dof and $60 \mathrm{~dB}$ in the vertical in agreement with previous measurements performed with a reduced load IP table ${ }^{9}$ and with a single GAS filter. Other experiments $[14,16]$ indicate that at least $20 \mathrm{~dB}$ of additional attenuation can be obtained implementing the subsystems foreseen for the compensation of the COP effects in both the IPs and GAS filters. ${ }^{10}$ Based on the measured transmissibilities, the plot in Fig. 15 was obtained. In this plot the displacement noise is estimated for the quieter seismic environment at the Livingston, LA site.

\section{Redundancy of the system}

Two accidental events during the commissioning revealed some significant aspects of SAS. Once, during installation, the cable of a horizontal actuator was severed. However, the system had enough redundancy and the commissioning was not interrupted, relying on the authority of the remaining three actuators.

In the second accident, the current in one of the actuator, because of a failure in one of the coil drivers, went out of control and started to oscillate rail to rail, dumping its maximum power on the coil. This accident lasted for a few days before being diagnosed but, though the in-vacuum coil must have heated up several degrees, it did not significantly over-heat and no outgassing was noticed in the UHV gages. During the time, the other three actuators acting on the same dofs under modal control, working against the oscillating one, maintained the table close to its required position, though producing a excessive displacement noise on the optics table.

Moreover, once, during the tests the hall air conditioning system failed for a few days. The temperature raised up to $30^{\circ} \mathrm{C}$. The higher temperature reduced the springs Young's modulus [34], eventually overwhelming the coil actuator authority and moving the table away from its nominal position. The actuators maintained the correct platform assets for a few degrees temperature excursion, much more than the fraction of a degree of thermal excursions that can be expected in an observatory experimental hall.

\section{Simulations}

Modeling and simulations accompanied the design phase of the system and the commissioning of the prototype.

A first kind of simulations studied the dependence of the internal modes of the system on the design shapes through Finite Element Modeling (FEA) [35]. The results guided the choice of materials and the configuration of the parts to avoid vibrational modes of the systems at frequencies below $100 \mathrm{~Hz}$. As a matter of fact the test on the prototype showed no significant resonances in the low frequency range due to the individual structure components.

Even though FEM simulations have been useful to study the internal modes of the rigid structure, they were unable to accurately reproduce the dynamic behavior of the complete

\footnotetext{
${ }^{9}$ The load was reduced by reducing the flex joint diameter to match the allowable payload of an existing horizontal shaking facility but keeping the same legs later used in the HAM-SAS system.

10 These subsystems, as well as the wire resonance damping O-rings, were not installed during the tests for lack of commissioning time.
} 

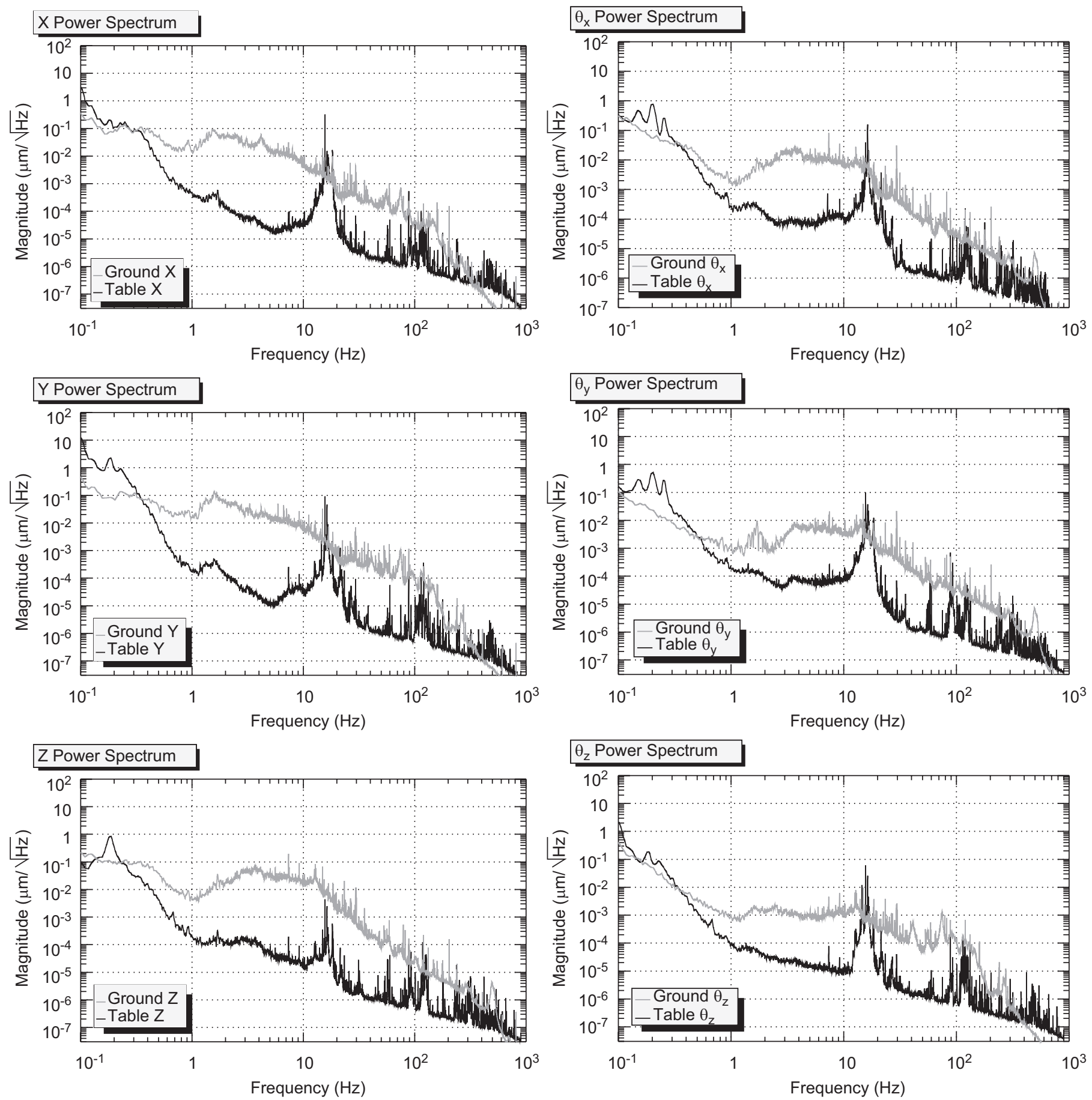

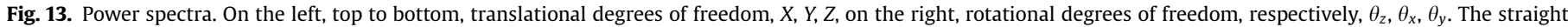

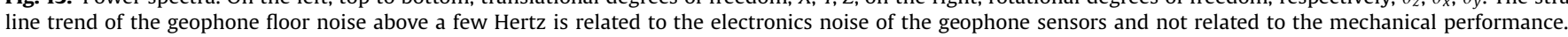

system, especially at low frequency. A simulation approach based on Multi-Body Dynamics (MBDyn) was explored. Two kinds of multibody models have been developed: a linear analytical model written in Maple symbolic language and a non-linear numerical model developed using a MBDyn simulation software [36]. In both models the angular stiffness of the top flexures of the IP were neglected and the dissipation mechanisms were taken into account using viscous damping to approximate structural/hysteretic damping. These simulations, once validated by comparison with a given tuning of the prototype's measured results, provide prediction of the performance for different system tunings.
The Maple model showed that with appropriate tuning of the IPs' counterweight and of the GAS blades' COP, more than $100 \mathrm{~dB}$ in attenuation can be achieved in the horizontal dofs and more than $80 \mathrm{~dB}$ in the vertical [37]. This model neglects factors like small misalignments of the spring box with respect to the horizontal plane or the uneven balancing of the mass on the optics table, and other effects of a not optimal tuning of the system which generate the cross-talk among the dofs observed in the actual prototype.

The MBDyn model has the advantage that it can easily include the system non-linearities and asymmetries. 

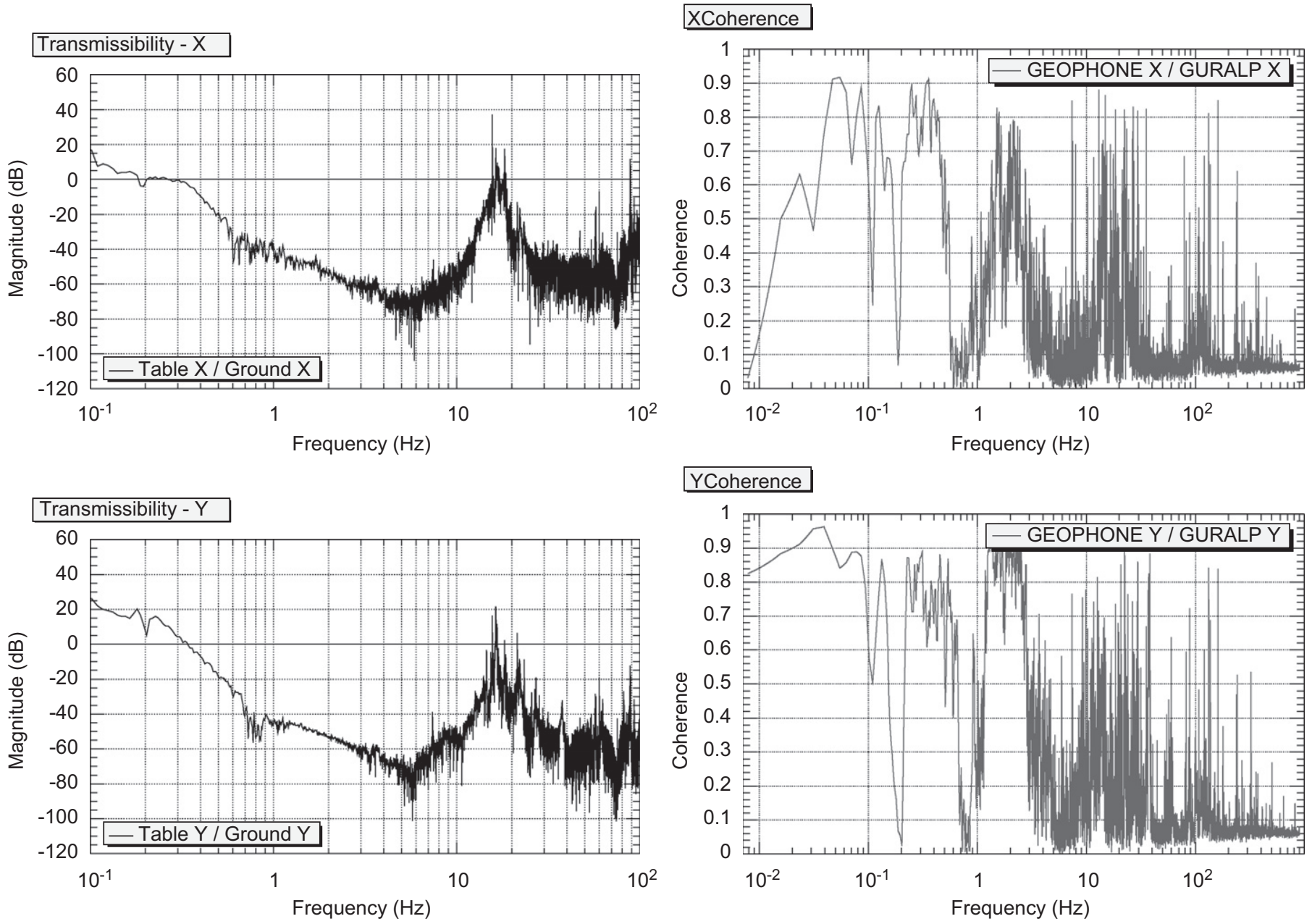

\section{YCoherence}
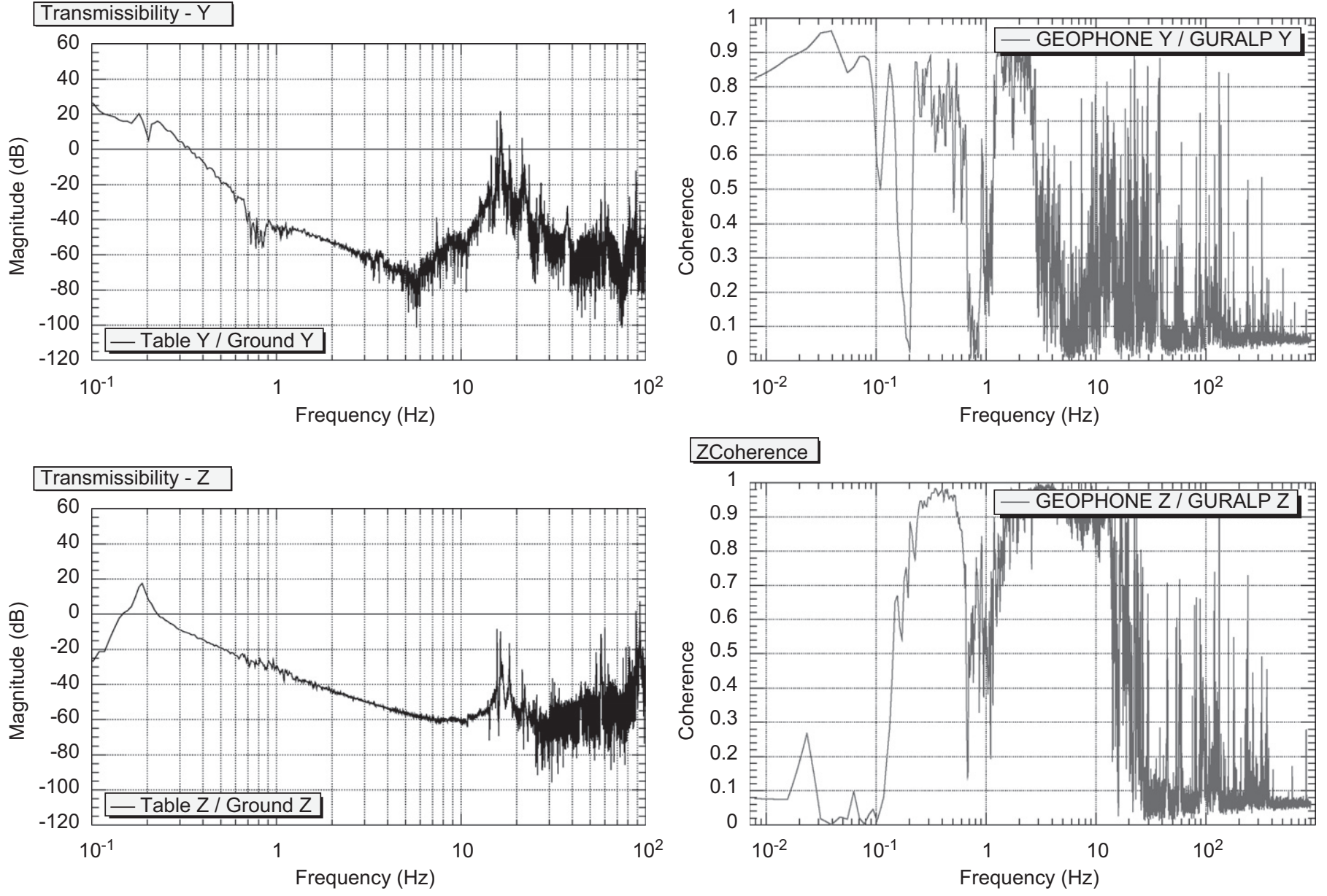

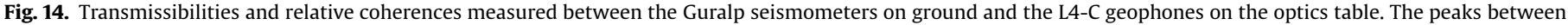
10 and $20 \mathrm{~Hz}$ are due to the undamped wire resonances,

Fig. 16 shows the transmissibilities resulting from these simulations compared with those measured for the three translational dofs $(X, Y, Z)$. Since MBDyn numerically analyzes the dynamics in the time domain, the frequency resolution of the simulated transfer function is inversely proportional to the number of seconds calculated (500 s in the plots), exactly as in a real measurement. Fitting the model to experimental data provides an estimate of the stiffness matrix of the system and of the compliances between the dofs. With this, one can predict the system performance with different tuning sets. For instance, Fig. 16 shows the seismic attenuation improvements when the modal resonant frequencies are further lowered respect with the experimental system setup. More than $60 \mathrm{~dB}$ would be achieved in vertical (still with no GAS Filter's COP compensation) and more than $100 \mathrm{~dB}$ in horizontal.

There was no opportunity to fully identify the shoulder at $250-300 \mathrm{mHz}$ in the horizontal response graphs in the course of the experimental trials. It might be due to an additional horizontal 


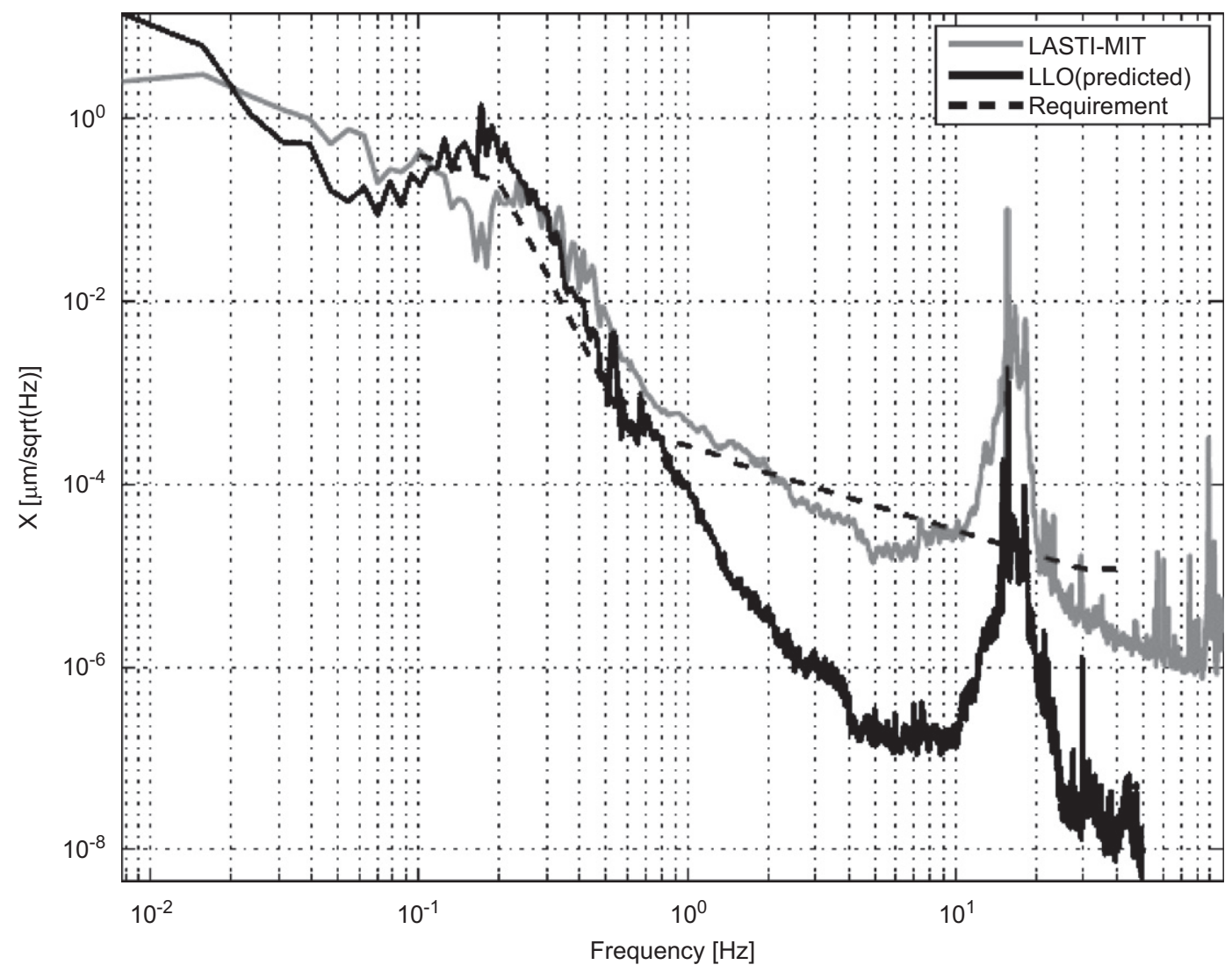

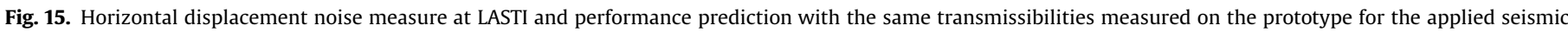
noise at the Livingston (Louisiana, USA) interferometer site. The dashed line represents the LIGO requirements.

resonance due to the GAS angular compliance, or a recoil effect of the tilt modes coupled to the horizontal direction by the overhanging load.

It ought to be reminded that the structure at $15-20 \mathrm{~Hz}$ in the data is due to the still undamped wire resonances (not included in the simulation). Above $1 \mathrm{~Hz}$, the distance of the measured transmissibilities from the simulated curve is mostly due to the fact that the latter does not include the sensors electronic noise (estimated of about $10^{-4} \mu \mathrm{m} / \sqrt{\mathrm{Hz}}$ at $1 \mathrm{~Hz}$ and scaling like $f^{-1}$ thereafter).

To study the response of the interaction of HAM-SAS with the suspended optics it supports, simulations at a higher complexity level was performed with the use of LIGOs end-to-end (E2E) simulation package [38]. Our particular interest was to assess the degree of the reaction force exerted by the Mode Cleaner (MC) triple suspensions back on the HAM-SAS platform, and to investigate its overall effect on the MCs performance. The E2E MC model consisted of the HAM-SAS component based on the Maple model which simulates the dynamics of HAM-SAS subsystems and the triple pendulum suspension component, Ref. [39] based on a Mathematica model, Ref. [40] which simulates the dynamics of the MC mirror. The triple suspension's damping control based on OSEM position sensors and the MC cavity's length sensing control were implemented. The backreaction effects via the local damping and length sensing control actuation forces were considered, in addition to the reaction force exerted by the suspension wires via the suspension point. Typical ground translational displacement in the direction of the X-arm was used as input to the HAM-SAS model. The correlation between the ground motions at HAM1 and HAM2 was also considered.
In Fig. 17, the effect of the back-reaction via the local damping actuation and the suspension point is assessed through comparison of the MC1 mirror's position motions computed with and without the back-reaction. In this run, the three MC mirrors were placed at the same locations as the Initial LIGO detector. Since the MC length control force is applied to MC2 on HAM2, the back-reaction associated with the MC length control is irrelevant in Fig. 17. Two cases are shown: with the only horizontal dof of HAM-SAS taken into account (labeled IP) and with both horizontal and vertical dofs taken into account (labeled IP and MGAS). When only the horizontal model is considered, the effect of back-reaction is negligible. However, when the back-reaction for the vertical model is included, the mirror's motion considerably increases in the $1-2 \mathrm{~Hz}$ range. In particular, the peak at $1.2 \mathrm{~Hz}$ becomes prominent, but the noise level still meets the requirement. This frequency is close to the triple suspension's mode \#5 resonance (described in Ref. [40]) which is dominated by the vertical motions of the middle mass and the lowest mass (the mirror). Fig. 17 indicates that the requirements are met from $50 \mathrm{mHz}$ up.

A feedback loop from the vertical inertial sensors, or even from the triple mirror position signal, to the table actuators, would counter the back-reaction effect and restore the attenuation performance of SAS. This correction would be useful during lock acquisition, when mirrors are actuated over macroscopic distances. The feasibility of inertial control of the platform was proved during the course of these tests by closing the feedback loop in all six dofs using the geophones as inertial sensors. As most of the detrimental effect is coupled via the tilts, just the three vertical dofs would be sufficient to mitigate the recoil effects. 

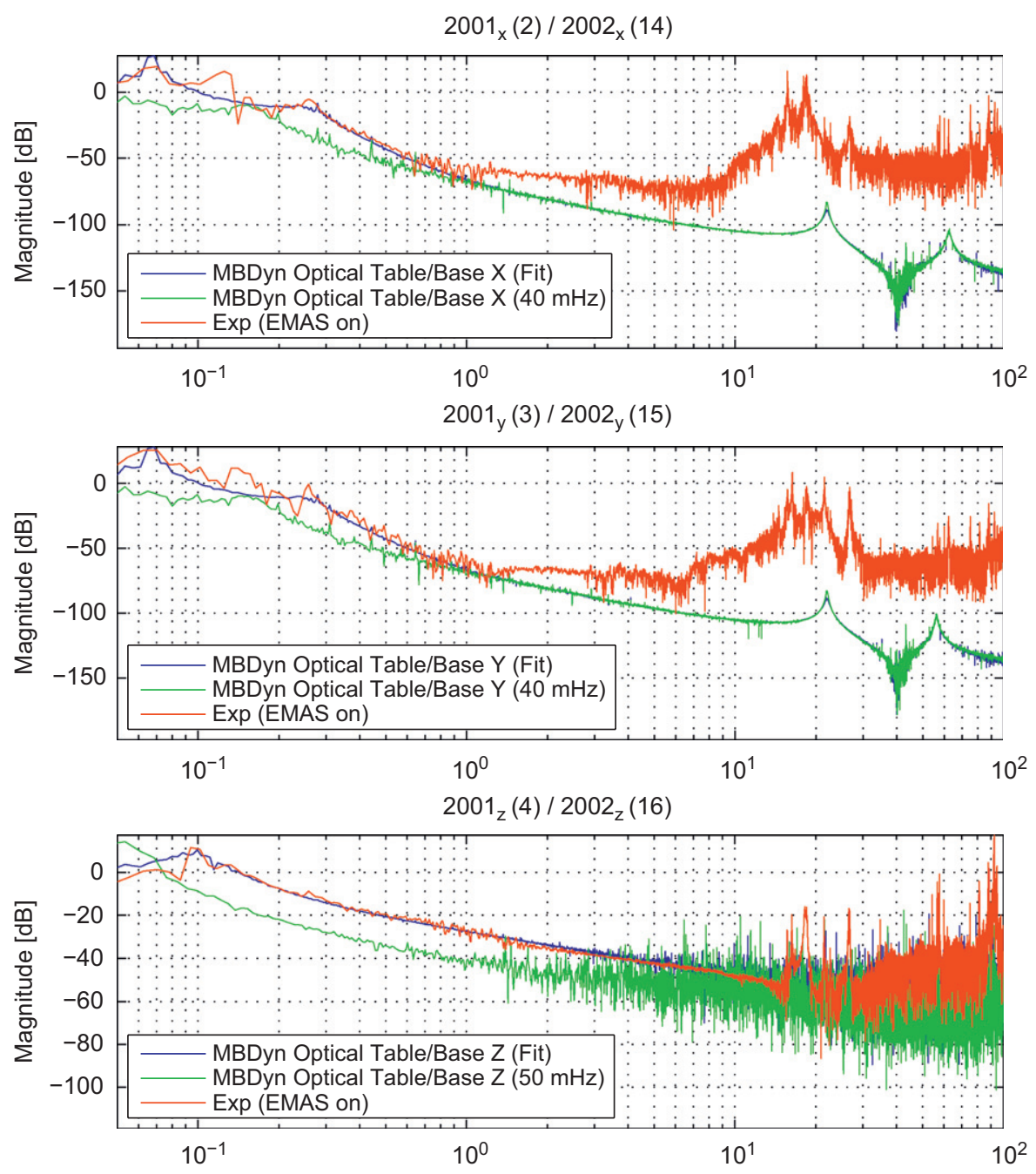

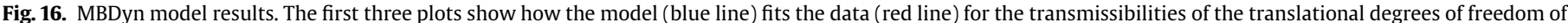

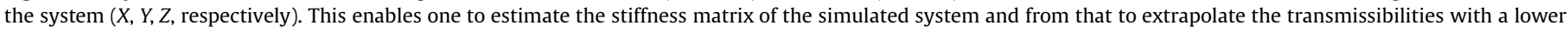

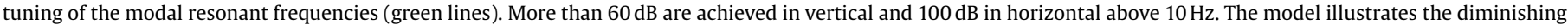

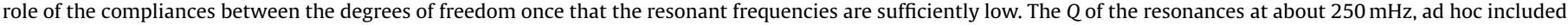
in the model to represent a compliance in the horizontal modes, is reduced when all the rigid body modes are tuned to lower frequencies.

With the E2E simulations it is also possible to predict the frequency noise of the MC transmitted light caused by MCs length fluctuation due to seismic disturbance. Note that this frequency noise is magnified by a factor of 250 (i.e. the ratio of the arm cavity length to the MC cavity length) in the arm cavity [41]. Fig. 18 shows the computed frequency noise of the MC transmitted light. When the back-reaction is included, the frequency noise increases in the entire studied frequency range of $0.1-10 \mathrm{~Hz}$, peaking at $1.2 \mathrm{~Hz}$. The back-reaction effect at $1.2 \mathrm{~Hz}$ dominates for the observed increase in the frequency noise. As a reference, the result of a computation using the Single Stage LIGO baseline HAM seismic isolation model before the back-reaction effect is taken into account is shown.

\section{Conclusions}

A first unit of SAS has been built, implemented and tested in a LIGO HAM vacuum chamber. Its passive attenuation performance achieved most of the LIGO requirements for HAM chambers, although the setup and tuning were only partially completed during the extremely short commissioning time [41,42]. The attenuation, with just preliminary tuning and no Center of
Percussion compensation, was such that the measurements were limited in most of the bandwidth by the sensitivity floor of the witness L4C geophones. The results from the simulations indicate that the complete fulfillment of the requirements would probably come with finer commissioning of the system and implementation of all its subsystems.

The system prototype achieved $60 \mathrm{~dB}$ of vertical attenuation and $70 \mathrm{~dB}$ horizontal attenuation. Static positioning controls proved effective also against large ambient thermal variations, and would be more than sufficient to compensate for tidal and other seasonal effects.

In terms of vacuum safety and operation reliability, HAM- SAS performances satisfied all expectations.

Due to the high quality and softness of its flexures, the frequency separation between the SAS rigid body modes and the internal resonances of its structures is very large. Its normal dynamical modes, and the voice coil actuators are, by design, oriented in the most favorable directions for controls. These characteristics make SAS a good platform for limited-bandwidth optional active controls, as verified by the inertial feedback tests performed using the witness geophone signals.

The SAS version of the seismic attenuation platform for the HAM chambers was realized with only 8 months intervening 


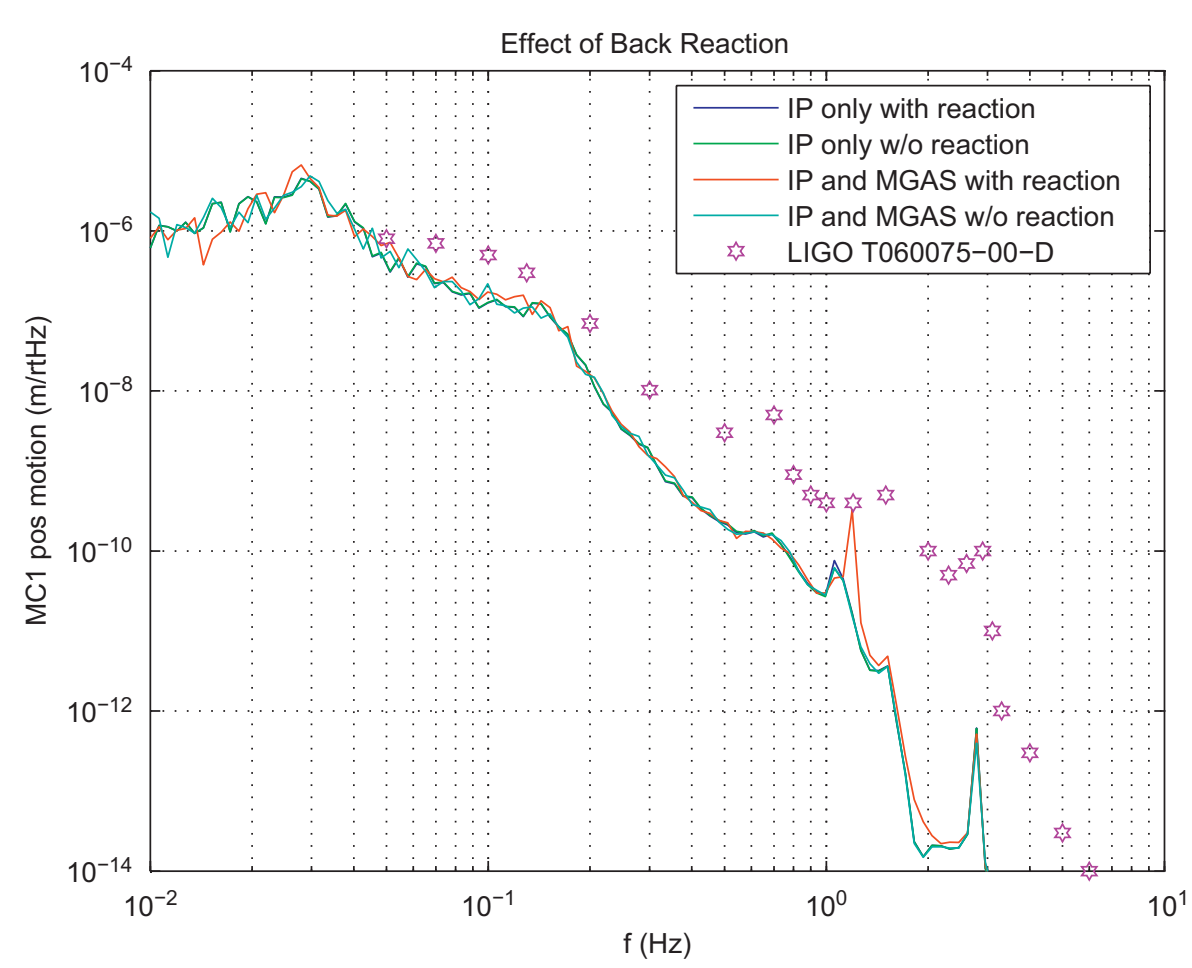

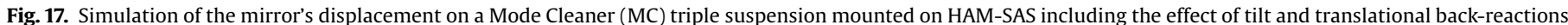
on the platform. The stars are the LIGO requirements.

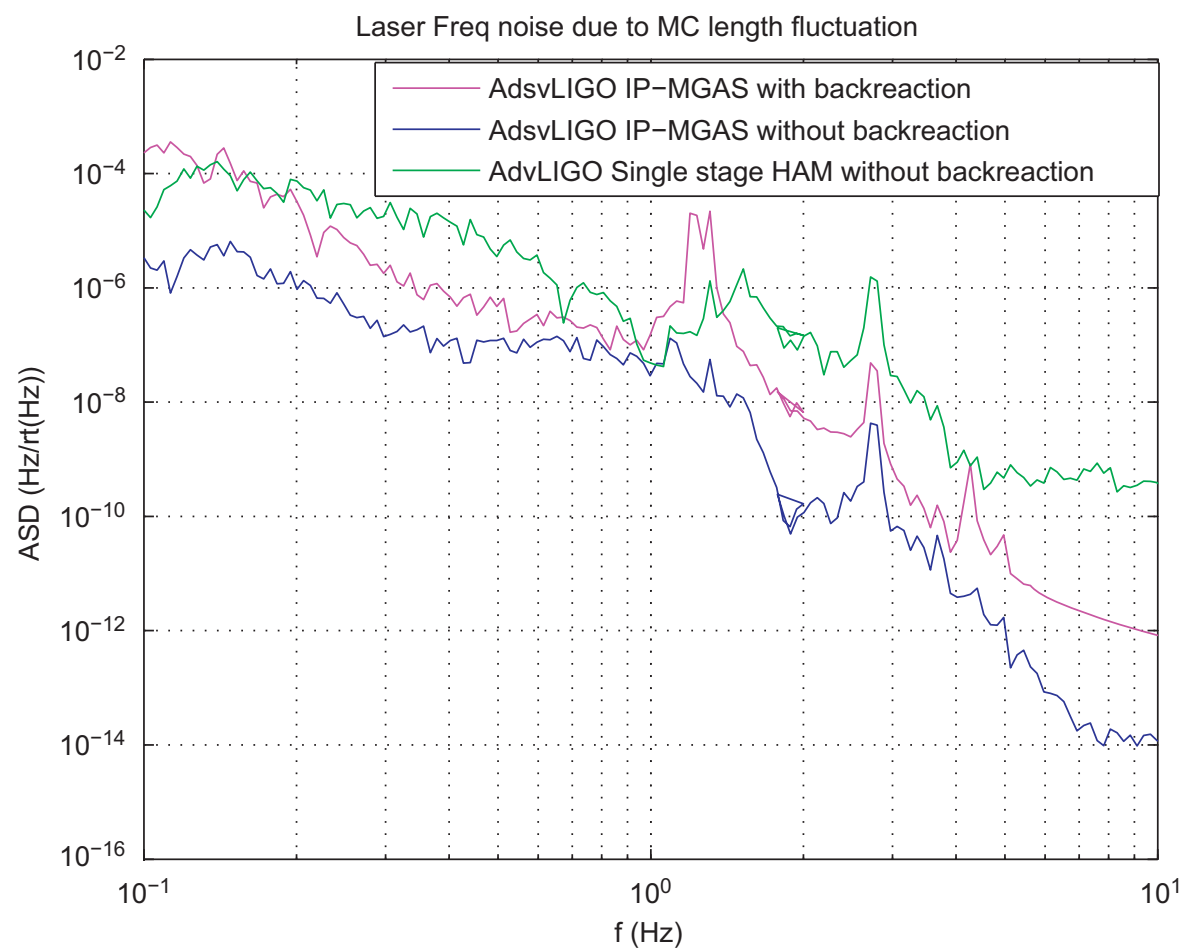

Fig. 18. Computed MC transmitted light's frequency noise caused by MC length fluctuation due to seismic disturbance.

between construction start and commissioning interruption. It weighs only $700 \mathrm{~kg}$, carrying $1000 \mathrm{~kg}$ of optics bench and payload. A SAS design suitable to instrument the BSC chambers was studied as well. The BSC configuration requires larger GAS filters (one of which has been prototyped in the course of the HAM-SAS tests) and longer IP legs, but it is simpler than the HAM version because its optics bench is suspended from wires below the SAS unit and is naturally stable with respect to tilts [43]. Additionally the $0.8 \mathrm{~m}$ wire pendulum stage that suspends the optics bench boosts the attenuation performance well above requirements. Apart from these considerations the HAM and BSC SAS design are topologically identical; therefore the results achieved with the HAM prototypes should be directly extensible to the BSC case. 
The SAS solution was not chosen for the seismic isolation in Advanced LIGO [44]. The experimental results obtained during the commissioning showed SAS as a viable solution to be successfully implemented in lieu of the Advanced LIGO baseline.

\section{Acknowledgements}

The authors thank Stan Whitcomb: he understands the problems addressed by this work. Without his support the HAM-SAS prototype would have never been built. Thanks are also due to Tullio Beneforti, Marcello Berchiolli, Maurizio Caturegli, Robert Laliberte, Myron MacInnis, Stefano Molesti, Mauro Morelli, Damiano Pescallini, Paolo Soldi. Their masterful and enthusiastic technical support made it possible to realize this very difficult project. Yumei Huang and Ilaria Taurasi also contributed with Finite Element Analysis of mechanical parts. The authors gratefully acknowledge the support of the United States National Science Foundation for the construction and operation of LIGO Interferometers. The LIGO observatories were constructed by the California Institute of Technology and Massachusetts Institute of Technology with funding from the National Science Foundation under cooperative agreement PHY 9210038. The LIGO Laboratory operates under cooperative agreement PHY-0107417.

\section{References}

[1] LIGO Scientific Collaboration, P. Fritschel (Ed.), Advanced LIGO Systems Design, LIGO Document T010075-00-D, 2001.

[2] A. Lazzarini, R. Weiss, LIGO Science Requirement Document, LIGO Document E950018-02 E, 1995.

[3] Advanced LIGO Team, Advanced LIGO Reference Design, LIGO Document 060056-08-M, 2007.

[4] N.A. Robertson, LIGO Collaboration, Seismic isolation and suspension systems for advanced, gravitational wave and particle astrophysics detectors, in: J. Hough, G. Sanders (Eds.), Proceedings of SPIE, vol. 5500, 2004, pp. 81-91.

[5] C.I. Torrie, Development of the suspensions for the GEO 600 gravitationa wave detector, Ph.D. Thesis, University of Glasgow, 2000.

[6] A. Bertolini, R. DeSalvo, C. Galli, G. Gennaro, M. Mantovani, S. Marka, V. Sannibale, A. Takamori, C. Torrie, Class. Quant. Grav. 23 (2006) S111.

[7] R. Abbott, R. Adhikari, G. Allen, S. Cowley, E. Daw, D. DeBra, J. Giaime, G. Hammond, M. Hammond, C. Hardham, J. How, W. Hua, W. Johnson, B. Lantz, K. Mason, R. Mittleman, J. Nichol, S. Richman, J. Rollins, D. Shoemaker, G. Stapfer, R. Stebbins, Class. Quant. Grav. 19 (7) (2002) 1591-1597.

[8] F. Acernese, et al., J. Phys. Conf. Ser., prepared for Tenth International Conference on Topics in Astroparticle and Underground Physics, Sendai, Japan, September 11-15, 2007, to be published.

[9] R. Takahashi, et al., Operational status of TAMA300 with the seismic attenuation system (SAS), in: Proceedings of the Amaldi, 2007.

[10] R. DeSalvo, et al., Nucl. Instr. and Meth. A 538 (2005) 526, LIGO-DCCG080243.

[11] R. DeSalvo, Passive, non-linear, mechanical structures for seismic attenuation, in: 5th ASME International Conference on Multibody Systems, Nonlinear Dynamics and Controls, Long Beach, CA, 24-28 September 2005, and references therein, LIGO-P050001-00-D.

[12] S. Braccini , F. Frasconi, et al., Meas. Sci. Technol. 11 (2000) 467. D051259-D.

[14] A. Stochino, The HAM-SAS seismic isolation system for the advanced LIGO gravitational wave detector, Master Thesis, University of Pisa, 2007. LIGO Document P070083-00-R.

[15] G. Losurdo, Ultra-low frequency inverted pendulum for the VIRGO test mass suspension, Ph.D. Thesis, Scuola Normale Superiore di Pisa-Classe di Scienze, Italy, October 1998.

[16] P. Raffai, et al., Inverted pendulum as low frequency pre-isolation for advanced gravitational wave detectors, LSC, submitted for publication.

[17] A. Takamori , et al., Class. Quant. Grav. 19 (2002) 1615.

[18] A. Stochino, R. DeSalvo, Y. Huang, V. Sannibale, Nucl. Instr. and Meth. A 580 (2007) 1559.

[19] A. Stochino, Performance improvement of the geometric anti spring (GAS) seismic filter for gravitational waves detectors, SURF-LIGO 2005 Final Report, P050074-00-R, 2005.

[20] C. Wang, et al., Nucl. Instr. and Meth. A 489 (1-3) (2002) 563.

[21] H. Tariq, et al., Nucl. Instr. and Meth. A 489 (2002) 570.

[22] F. Acernese, et al., Phys. Rev. D, in preparation.

[23] R. Takahashi, et al., Control system for the seismic attenuation system (SAS) in TAMA300, in: Proceedings of the Amaldi, 2007.

[24] D. Coyne, Earthquake risk and recovery: lessons from the 2/28/01 Olympia, WA quake, LIGO Document G010208-00-D, 2001.

[25] E. Majorana, Mirror suspension control VSR1 learning, LSC-Virgo Meeting at Hannover, October 25, 2007, LIGO Document G070744-00-Z, 2007.

[26] N. Virdone, et al., Nucl. Instr. and Meth. 593 (3) (2008) 597.

[27] A. Bertolini, High sensitivity accelerometers for gravity experiments, Ph.D. Thesis, University of Pisa, LIGO Document P010009-00-Z, 2001.

[28] H. Goldstein, Classical Mechanics, Addison-Wesley, Reading, MA, 2001.

[29] A. Stochino, The HAM-SAS seismic isolation system for the advanced LIGO interferometers, in: Caltech LIGO Seminar Presentation, Pasadena, September 4, 2007, LIGO Document G050484-00, 2007.

[30] R. Bork, LASTI digital control and data system design, February 3, 2006, LIGO Document T060031-00-C, 2006.

[31] J. Heefner, LASTI HAM-SAS digital controls requirements and design overview, in: Presentation, Caltech, Pasadena, November 22, 2006, LIGO Document G060596-00-C, 2006.

[32] M. Mantovani , R. DeSalvo, Nucl. Instr. and Meth. A 554 (1-3) (2005) 546.

[33] H.S. Bae, Active vibration isolation and alignment issues for LIGO, Masters Thesis, Department of Mechanical Engineering, Stanford University, August 1999.

[34] N. Verdone, et al., Extended-time-scale creep measurement on maraging cantilever blade springs, LIGO Document P070095-00-Z, 2007.

[35] V. Boschi, et al., HAM-SAS spring box simulations, LIGO Document T06006600-E, 2006.

[36] V. Boschi, HAM-SAS Mechanics: Status of Modeling, in: LSC Meeting, August 14-17th, 2006, Louisiana State University, LIGO Document G060445-00-R, 2006.

[37] V. Boschi, et al., AdLIGO HAM-SAS mechanical model with lumped elements, LIGO Document T060037-00-R, 2006.

[38] M. Barton, B. Bhawal, M. Evans, H. Yamamoto, S. Yoshida, J. Phys. Conf. Ser. 32 (2006) 398.

[39] N.A. Robertson, et al., Advanced LIGO suspension system conceptual design, LIGO Document T010103-05-D, 2006.

[40] M. Barton, Models of the advanced LIGO suspensions in MathematicaTM, LIGO Document T000051-01-D, 2005.

[41] P. Fritschel, HAM seismic isolation requirements, LIGO Document T060075-D$00,2006$.

[42] D. Ottaway, HAM SAS test plan at LASTI, LIGO Document T060213-02-R, 2006.

[43] V. Sannibale, et al., Recent results of a seismically isolated optical table prototype designed for advanced LIGO, in: Proceedings of the Amaldi 2007, LIGO Document P070127-01-Z, 2007.

[44] D. Shoemaker, Advanced LIGO HAM seismic isolation technology: consideration of HAM-SAS as alternative to the baseline design, LIGO Document M070057-00-M, 2007. 